\title{
A TWO-EQUATION HEAT TRANSFER MODEL FOR WALL TURBULENT SHEAR FLOWS
}

\author{
M. S. Youssef \\ Mechanical Engineering Department, Faculty of Engineering, Assiut \\ University, Assiut, Egypt.
}

(Received February 8, 2006 Accepted August 21, 2006)

A proposal for closing the Reynolds-averaged energy equation is presented at the two-equation level of turbulence modeling. The eddy diffusivity for heat is proposed as a function of the local energy of turbulence, $k$, and the local temperature time scale, $\tau_{t}$, instead of using mixed time scale, $\tau_{m}$. The proposed two-equation heat transfer model solves two differential equations, one for the temperature variance, $k_{t}$, and the other for the temperature time scale, $\tau_{t}$. The near-wall limiting behavior of turbulent quantities associated with heat transfer has been captured with the proposed model. Therefore, an additional term is included in the temperature variance equation to improve the prediction of near-wall behavior. Moreover, an exact and noval equation for the temperature time scale, $\tau_{t}$, is introduced in this study. The proposed $k_{t}-\tau_{t}$ heat transfer model does not suffer from numerical stiffness problems since natural boundary conditions for the variables $k_{t}$ and $\tau_{t}$ are used $\left(k_{t}=\tau_{t}=0\right.$ at $\left.y=0\right)$. The proposed model is assessed by application to fully-developed turbulent channel flows under different wall thermal conditions with different values of Reynolds numbers. The results for all cases examined showed good agreement with those of the direct numerical simulation data.

KEYWORDS: Turbulent Flow, $k-\varepsilon$ Model, $k-k L$ Model, $k_{t}-\varepsilon_{t}$ Model, Fully-Developed Turbulent Channel Flow, Wall Thermal Conditions.

\section{NOMENCLATURE}

\footnotetext{
$\mathrm{A}_{\lambda}, \mathrm{B}_{\lambda}$ : temperature field model constants

$\mathrm{C}_{\mathrm{D} 1}$ : temperature field model constant

$\mathrm{C}_{\mathrm{P} 1}$ : temperature field model constant

$\mathrm{C}_{\mathrm{W}}$ : velocity field model function
}

$\mathrm{C}_{\mathrm{D}}, \mathrm{C}_{\mathrm{P}}, \mathrm{C}_{\mathrm{T}}$ : velocity field model constants $\mathrm{C}_{\mathrm{D} 2}$ : temperature field model constant $\mathrm{C}_{\mathrm{P} 2}$ : temperature field model constant $\mathrm{C}_{\lambda}$ : temperature field model constant 
$c_{p}:$ specific heat at constant pressure

$\mathrm{f}_{\mathrm{wkt}}$ : temperature field model function

$f_{D 2}$ : temperature field model function

$\mathrm{f}_{\mathrm{P} 2}$ : temperature field model function

$\mathrm{f}_{\lambda}$ : temperature field model function

$\mathrm{k}$ : turbulence kinetic energy

$\mathrm{L}$ : turbulence length scale

Pr : molecular Prandtl number

$\mathrm{q}_{\mathrm{w}}$ : wall heat flux

$\mathrm{t}:$ temperature fluctuation or time

$\mathrm{U}:$ mean velocity in $\mathrm{x}$ direction

$U_{\varepsilon}:$ Kolmogorov velocity scale

$\mathrm{u}_{\tau}$ : friction velocity, $\sqrt{\tau_{w} / \rho}$

$\mathrm{v}$ : fluctuating velocity in y direction

$\mathrm{y}:$ distance from the wall $\mathrm{f}_{\mathrm{w}}$ : velocity field model function

$f_{D 1}$ : temperature field model function

$\mathrm{f}_{\mathrm{P} 1}$ : temperature field model function

$\mathrm{f}_{\mu}$ : velocity field model function

$\mathrm{h}$ : channel half-width

$k_{t}$ : temperature variance, $\overline{t^{2}} / 2$

$\mathrm{P}, \mathrm{p}:$ mean and fluctuating pressures

$\operatorname{Pr}_{\mathrm{t}}$ : turbulent Prandtl number

$\bar{T}$ : mean temperature

$t_{r}$ : friction temperature, $q_{w} / \rho c_{p} u_{r}$

$U_{m}$ : mean bulk velocity

$\mathrm{u}$ : fluctuating velocity in $\mathrm{x}$ direction

$\mathrm{V}:$ mean velocity in y direction

$\mathrm{x}$ : stream-wise coordinate

$y^{*}$ : dimensionless distance from wall.

\section{Greek Symbols}

$\alpha, \alpha_{t}$ : molecular and eddy diffusivities for heat

$\varepsilon$ : dissipation rate of $\mathrm{k}$

$\varepsilon_{\mathrm{t}}$ : dissipation rate of $\mathrm{k}_{\mathrm{t}}$

$\theta:$ momentum thickness of boundary layer

$v, v_{\mathrm{t}}:$ molecular and eddy viscosities

$\rho:$ density

$\sigma_{\mathrm{k}}, \sigma_{\mathrm{kL}}$ : velocity field model constants

$\sigma_{\mathrm{h}}, \sigma_{\tau 1}, \sigma_{\tau 2}$ : temperature field model constants

$\tau_{\mathrm{m}}:$ mixed time scale, $\sqrt{\tau_{u} \tau_{t}}$

$\tau_{\mathrm{u}}, \tau_{\mathrm{t}}$ : time scales of velocity and temperature fields, $k / \varepsilon, k_{t} / \varepsilon_{t}$, respectively

$\tau_{\mathrm{w}}:$ wall shear stress.

\section{Dimensionless Parameters}

$\operatorname{Re}_{\tau}$ : channel flow Reynolds number, $u_{\tau} h / v$

Re : Reynolds number, $2 U_{m} h / v$

$\mathrm{R}_{\mathrm{t}}$ : turbulence Reynolds number, $\sqrt{k} L / v$

$\mathrm{R}_{\mathrm{h}}$ : turbulence Reynolds number, $k \tau_{t} / v$

$\operatorname{Re}_{\theta}$ : momentum thickness Reynolds number for boundary layer, $U_{e} \theta / v$

\section{Subscripts}

e : boundary layer edge

w : wall value 


\section{Superscripts}

()$^{+}$: normalization by wall variables, i.e., $\mathrm{u}_{\tau}$ for mean velocity, $\mathrm{u}_{\tau}{ }^{2}$ for $\mathrm{k}$ or $\overline{u v}, v / \mathrm{u}_{\tau}$ for $\mathrm{L}$ or $\mathrm{y}, \mathrm{u}_{\tau}{ }_{\tau}^{4} / v$ for dissipation rate of $\mathrm{k}, v / \mathrm{u}_{\tau}{ }^{2}$ for $\tau_{\mathrm{u}}$ or $\tau_{\mathrm{t}}, \mathrm{t}_{\tau}$ for $\bar{T}, t_{\tau}^{2}$ for $k_{t}$, and $u_{\tau}^{2} t_{\tau}^{2} / v$ for dissipation rate of $k_{t}$.

$\overline{(\tau)}$ : time mean value

\section{INTRODUCTION}

The turbulence model for heat transfer is a set of differential equations which, when solved with the mean-flow and turbulence Reynolds stresses equations, allow calculations of relevant correlations and parameters that simulate the behaviour of thermal turbulent flows. Like the classification of turbulence models for the Reynolds stresses, the phenomenological turbulent heat transfer models are clssified into zeroequation, two-equation, and heat-flux equation models.

The zero-equation heat transfer model is a typical and most conventional method for analyzing the turbulent heat transfer, in which the eddy diffusivity for heat $\alpha_{t}$ is prescribed via the known eddy viscosity $v_{t}$ together with the most probable turbulent Prandtl number $\operatorname{Pr}_{t}$, so that $\alpha_{t}=v_{t} / \operatorname{Pr}_{t}$ [1-3]. Thus, in this formulation the analogy is assumed tacitly between turbulent heat and momentum transfer and the turbulent Prandtl number $\operatorname{Pr}_{t}$ needs to be prescribed. However, shear flow measurements [4,5] and direct simulation data [6,7] showed that an analogy between heat and momentum transfer as represented by a constant turbulent Prandtl number could not adequately reflect the physical phenomenon of heat transport, even for simple wall shear flows. Furthermore, these data [4-7] showed that the turbulent Prandtl number $\mathrm{Pr}_{\mathrm{t}}$, instead of being constant, increased towards a wall. Its value at the wall was determined to be about 1.1 and exceeded the $0.7-0.9$ value normally assumed for wall shear flow calculations. In other words, there are so many ambiguous points in $\operatorname{Pr}_{t}$ itself, and none of the empirical formulae for $\operatorname{Pr}_{t}$ can work universally [8].

The heat-flux equation model ought to be more universal, at least in principle. In this model, however, correct modelling of the scalar-pressure gradient correlation term and the dissipation term is generally critical in obtaining correct heat flux values $[9,10]$. Efforts are directed toward developing new models, especially of the scalarpressure gradient correlation term to improve the overall accuracy of existing models. Although the developed models demonstrated improvements in thin shear flows, they sometimes gave poor predictions compared to those obtained by the previous models in general cases $[11,12]$. Thus, the heat-flux equation model needs further study and refinements.

Two-equation $k_{t}-\varepsilon_{t}$ heat transfer models have been improved [1, 2, 13-18] since Nagano and Kim [3] proposed the first model for wall turbulent shear flows. A common feature of these models, however, is that damping functions to take account of the wall blocking on temperature fluctuations are invoked in their formulations. 
However, many important technological applications require the integration of heat transfer models directly to a solid boundary, particularly in problems where wall transport properties are needed. It was established that the two-equation heat transfer model is a powerful tool for predicting the heat transfer in flows with almost complete dissimilarity between velocity and thermal fields [13-18]. Two-equation $k_{t}-\varepsilon_{t}$ heat transfer model has major problem associated with it. It arises from the lack of natural boundary conditions for the temperature variance dissipation rate $\varepsilon_{t}$, which has caused modelers to use a boundary condition results numerical stiffness in calculations (the boundary condition that ties $\varepsilon_{t}$ to higher-order derivatives of the temperature variance $k_{t}$ [1, 2, 13-17]). It should be mentioned here that, such problems of boundary conditions are also associated with the near-wall $k-\varepsilon$ model for velocity field $[19,20]$. Moreover, another defect in all two-equation heat transfer models published in the literature is the characteristic time scale used in evaluating the eddy diffusivity for heat $\alpha_{t}$. All these models used the mixed time scale $\tau_{m}$, which is almost equal to the geometric mean of $\tau_{u}$ and $\tau_{t}$ in simple shear layers $\left(\tau_{m}=\sqrt{\tau_{u} \tau_{t}}\right)$. Since the interactions between momentum and heat transport are already included in the characteristic velocity scale, an appropriate time scale would be only given by the scalar time scale $\tau_{t}$. Therefore, models along alternative lines continue to be proposed.

Problems associated with the published $k_{t}-\varepsilon_{t}$ models can be largely remedied by solving a modeled transport equation for the scalar time scale $\tau_{t}$. The reason for this choice is attributed not only to the natural boundary condition for $\tau_{t}$ ( $\tau_{t}=0$ at the wall) but also to the balance of terms at the wall in the modeled transport equation for $\tau_{t}$. These features are primarily responsible for the development of a new heat transfer model having more computationally robust performance.

In the present paper, the author attempts to construct a new reliable $k_{t}-\tau_{t}$ turbulence heat transfer model using direct numerical simulation (DNS) databases for wall turbulence with heat transfer. In this model, a noval exact transport equation of $\tau_{t}$ is derived based on the exact transport equations for $k_{t}$ and $\varepsilon_{t}$. Therefore, the eddy diffusivity for heat, $\alpha_{t}$, can be determined from the solutions of transport equations for $k_{t}$ and $\tau_{t}$. The near-wall behavior of heat transfer turbulence is accurately incorporated in the present model. Meanwhile, the model should not suffer from numerical instabilities and should be capable of predicting the turbulent heat transfer over a wide range of Reynolds number. The present model is evaluated through its application to fully-developed turbulent channel flow with heat transfer at low Reynolds number under different wall thermal conditions. Comparisons are 
made with the predictions of other published $k_{t}-\varepsilon_{t}$ models to assess the present model performance.

\section{TWO-EQUATION MODEL FOR THE VELOCITY FIELD}

Recently, Youssef [21] proposed a new k-kL two-equation model of turbulence for velocity field which reproduces the correct near-wall asymptotic relations of turbulence. This $\mathrm{k}-\mathrm{kL}$ model has been assessed by application to fullydeveloped turbulent channel flows at different Reynolds numbers and flat-plate boundary layer flow. Therefore, for the calculation of velocity field in the present study, k-kL model of Youssef [21] will be used in which the following governing equations may be rewritten as follows:

$$
\begin{gathered}
\frac{\partial U_{i}}{\partial x_{i}}=0 \\
\frac{D U_{i}}{D t}=-\frac{\partial P}{\partial x_{i}}+\frac{\partial}{\partial x_{j}}\left\{v\left(\frac{\partial U_{i}}{\partial x_{j}}+\frac{\partial U_{j}}{\partial x_{i}}\right)-\overline{u_{i} u_{j}}\right\} \\
\left.\frac{D k}{D t}=\frac{\partial}{\partial x_{j}}\left[\left(v+\frac{v_{t}}{\sigma_{k}}\right) \frac{\partial k}{\partial x_{j}}\right]-\frac{\partial U_{i}}{u_{i} u_{j}} \frac{\partial x_{j}}{D k L}=\frac{\left.C_{D} \frac{k^{1.5}}{L}+2 v \mathrm{f}_{\mathrm{w}} \frac{k}{x_{2}^{2}}\right]}{\partial x_{j}}\left[\left(v+\frac{v_{t}}{\sigma_{k L}}\right) \frac{\partial k L}{\partial x_{j}}\right]-C_{P} \mathrm{~L} \frac{\partial U_{i} u_{j}}{\partial x_{j}}\right)-\left[C_{T}+\mathrm{C}_{\mathrm{W}}\right] \mathrm{k}^{1.5}
\end{gathered}
$$

with

$$
-\overline{u_{i} u_{j}}=v_{t}\left(\frac{\partial U_{i}}{\partial x_{j}}+\frac{\partial U_{j}}{\partial x_{i}}\right)-\frac{2}{3} k \delta_{i j}
$$

and

$$
v_{t}=f_{\mu} \sqrt{k} L
$$

\begin{tabular}{|c|c|c|c|c|}
\hline$\sigma_{\mathrm{k}}$ & $\mathrm{C}_{\mathrm{D}}$ & $\sigma_{\mathrm{kL}}$ & $\mathrm{C}_{\mathrm{P}}$ & $\mathrm{C}_{\mathrm{T}}$ \\
\hline 1.2 & 0.1 & 1.2 & 0.77 & 0.055 \\
\hline$f_{w}$ & \multicolumn{4}{|c|}{$\exp \left[-\left(\mathrm{y}^{+} / 12\right)^{0.5}\right]$} \\
\hline $\mathrm{f}_{\mu}$ & \multicolumn{4}{|c|}{$\left[1-\exp \left(-\mathrm{y}^{+} / 26.5\right)\right] \times\left[1-\exp \left(-\mathrm{y}^{+} / 45\right)\right] \times\left\{1+1.25 \exp \left[-\left(\mathrm{R}_{\mathrm{t}} / 0.05\right)^{2}\right] / \mathrm{R}_{\mathrm{t}}^{0.5}\right\}$} \\
\hline $\mathrm{C}_{\mathrm{W}}$ & \multicolumn{4}{|c|}{$1.8\left[1+1.5 \exp \left(-\mathrm{y}^{+} / 45\right)\right](\mathrm{L} / \mathrm{y})^{5}$} \\
\hline
\end{tabular}

where $D / D t$ implies the substantial derivative. In Eq. (3), $x_{2}$ represents the distance normal to the wall. The constants and functions in the used $\mathrm{k}-\mathrm{kL}$ model are summarized in Table 1 [21].

Table 1. Constants and functions in the used $\mathrm{k}-\mathrm{kL}$ model. 


\section{TWO-EQUATION MODEL FOR THE THERMAL FIELD}

A temperature field can be obtained by solving the energy equation. When temperature is regarded as a passive scalar, the energy equation is expressed as follows [3]:

$$
\frac{D \bar{T}}{D t}=\frac{\partial}{\partial x_{j}}\left(\alpha \frac{\partial \bar{T}}{\partial x_{j}}-\overline{u_{j} t}\right)
$$

However, Eq. (7) is not closed since it contains an unknown turbulent heat flux $\overline{u_{j} t}$. The turbulent heat flux $\overline{u_{j} t}$ is described using the concept of eddy diffusivity for heat $\alpha_{t}$ by the following simple gradient form [3]:

$$
-\overline{u_{j} t}=\alpha_{t} \frac{\partial \bar{T}}{\partial x_{j}}
$$

In a mannar similar to that used in defining turbulent eddy viscosity $v_{t}$, the eddy diffusivity for heat $\alpha_{t}$ is expressed as a function of the state of velocity and temperature fields. Dimensionally, $\alpha_{t}$ is the product of a velocity scale and a length scale. A characteristic velocity scale for turbulent flow is $k^{1 / 2}$. If the interactions between momentum and heat transport are to be modeled properly, an approperiate length scale would be given by multiplation of the velocity scale, $k^{1 / 2}$, and a time scale. Generally, the time scale is considered one of three options, namely, the time scale of thermal field, the time scale of velocity field, and the geometric mean of both. Therefore, it is interpreted that $\alpha_{t} \alpha k \tau$ where $\tau$ is the characteristic time scale. The time scale of the thermal field $\tau_{t}$ can be evaluated from the temperature variance $k_{t}$ and its dissipation rate $\varepsilon_{t}$, while the time scale for the velocity field is determined from $k$ and its dissipation rate $\varepsilon$. In the proposed model, the scalar time scale $\tau_{t}$ is adopted in calculating the eddy diffusivity for heat. Hence, $\alpha_{t}$ may be modeled as:

$$
\alpha_{t}=C_{\lambda} f_{\lambda} k \tau_{t}
$$

where $C_{\lambda}$ is the model constant, and $f_{\lambda}$ is the model function, which has some properties in common with $f_{\mu}$ in equation (6), as will be described later. In Eq. (9), the time scale of the thermal field $\tau_{t}$ is obtained from a new differential equation. The exact transport equations for $k_{t}$ and $\varepsilon_{t}$ are expressed symbolically as follows [16]:

$$
\frac{D k_{t}}{D t}=D_{k t}+T_{k t}+P_{k t}-\varepsilon_{t}
$$




$$
\frac{D \varepsilon_{t}}{D t}=D_{\varepsilon t}+T_{\varepsilon t}+P_{\varepsilon t}^{1}+P_{\varepsilon t}^{2}+P_{\varepsilon t}^{3}+P_{\varepsilon t}^{4}-\Psi_{\varepsilon t}
$$

The different terms on right-hand sides in Eqs. (10) and (11) are identified as:

Molecular diffusion of $k_{t}$ :

$$
D_{k t}=\alpha \frac{\partial^{2} k_{t}}{\partial x_{j} \partial x_{j}}
$$

Turbulent diffusion of $k_{t}$ :

$$
T_{k t}=-\frac{\partial \overline{u_{j} k_{t}^{\prime}}}{\partial x_{j}}
$$

Mean gradient production of $k_{t}$ :

$$
P_{k t}=-\overline{u_{j} t} \frac{\partial \bar{T}}{\partial x_{j}}
$$

Molecular diffusion of $\varepsilon_{t}$

$$
D_{\varepsilon t}=\alpha \frac{\partial^{2} \varepsilon_{t}}{\partial x_{j} \partial x_{j}}
$$

Turbulent diffusion of $\varepsilon_{t}$ :

$$
T_{\varepsilon t}=-\frac{\partial \overline{u_{j} \varepsilon_{t}^{\prime}}}{\partial x_{j}}
$$

Mean gradient production of $\varepsilon_{t}$ :

$$
\begin{aligned}
& P_{\varepsilon t}^{1}=-2 \alpha \overline{\frac{\partial u_{j}}{\partial x_{k}} \frac{\partial t}{\partial x_{k}}} \frac{\partial \bar{T}}{\partial x_{j}} \\
& P_{\varepsilon t}^{2}=-2 \alpha \overline{\frac{\partial t}{\partial x_{k}} \frac{\partial t}{\partial x_{j}} \frac{\partial U_{j}}{\partial x_{k}}}
\end{aligned}
$$

Gradient production of $\varepsilon_{t}$ :

$$
P_{\varepsilon t}^{3}=-2 \alpha \overline{u_{j} \frac{\partial t}{\partial x_{k}}} \frac{\partial^{2} \bar{T}}{\partial x_{j} \partial x_{k}}
$$

Turbulent production of $\varepsilon_{t}$ :

$$
P_{\varepsilon t}^{4}=-2 \alpha \overline{\frac{\partial u_{j}}{\partial x_{k}} \frac{\partial t}{\partial x_{k}} \frac{\partial t}{\partial x_{j}}}
$$


Destruction of $\varepsilon_{t}$ :

$$
\Psi_{\varepsilon t}=2 \alpha^{2} \overline{\left(\frac{\partial^{2} t}{\partial x_{k} \partial x_{j}}\right)^{2}}
$$

where $k_{t}^{\prime}=t^{2} / 2$ and $\varepsilon_{t}^{\prime}=\alpha\left(\partial t / \partial x_{k}\right)^{2}$

From basic definition of $\tau_{t}=k_{t} / \varepsilon_{t}$, one could derive the exact transport equation for $\tau_{t}$ as follows:

$$
\frac{D \tau_{t}}{D t}=\frac{\tau_{t}}{k_{t}}\left(\frac{D k_{t}}{D t}\right)-\frac{\tau_{t}^{2}}{k_{t}}\left(\frac{D \varepsilon_{t}}{D t}\right)
$$

Substitution from Eqs. (10) and (11) in Eq. (22), we finally obtain

$$
\begin{aligned}
\frac{D \tau_{t}}{D t}= & \frac{\tau_{t}}{k_{t}} D_{k t}+\frac{\tau_{t}}{k_{t}} T_{k t}+\frac{\tau_{t}}{k_{t}} P_{k t}-1 \\
& \quad-\frac{\tau_{t}^{2}}{k_{t}} D_{\varepsilon t}-\frac{\tau_{t}^{2}}{k_{t}}\left[T_{\varepsilon t}+P_{\varepsilon t}^{1}+P_{\varepsilon t}^{2}+P_{\varepsilon t}^{3}+P_{\varepsilon t}^{4}-\Psi_{\varepsilon t}\right]
\end{aligned}
$$

\section{MODELING OF $\tau_{t}$ EQUATION}

The turbulent diffusion term of $k_{t}, T_{k t}$, is usually modeled by using the gradient-type diffusion model as follows:

$$
T_{k t}=\frac{\partial}{\partial x_{j}}\left[\frac{\alpha_{t}}{\sigma_{h}} \frac{\partial k_{t}}{\partial x_{j}}\right]
$$

therefore, the term $\tau_{t} T_{k t} / k_{t}$ in the righ-hand side of Eq. (23) is further approximated to yields

$$
\frac{\tau_{t}}{k_{t}} T_{k t}=\frac{\tau_{t}}{k_{t}} \frac{\partial}{\partial x_{j}}\left[\frac{\alpha_{t}}{\sigma_{h}} \frac{\partial k_{t}}{\partial x_{j}}\right]=\frac{\partial}{\partial x_{j}}\left[\frac{\alpha_{t}}{\sigma_{h}} \frac{\partial \tau_{t}}{\partial x_{j}}\right]
$$

It should be mentioned here that the molecular diffusion of $k_{t}$ need not to be modeled, while, and the mean production term of $k_{t}$ in Eq. (14) may be rewritten after substitution for the turbulent heat flux $\overline{u_{j} t}$, Eq. (8), as follows: 


$$
P_{k t}=-\overline{u_{j} t} \frac{\partial \bar{T}}{\partial x_{j}}=\alpha_{t}\left(\frac{\partial \bar{T}}{\partial x_{j}}\right)^{2}
$$

The molecular diffusion of $\varepsilon_{t}$ in Eq. (15) is rewritten as:

$$
D_{\varepsilon t}=\alpha \frac{\partial^{2} \varepsilon_{t}}{\partial x_{j} \partial x_{j}}=\alpha \frac{\partial^{2}}{\partial x_{j} \partial x_{j}}\left(\frac{k_{t}}{\tau_{t}}\right)
$$

Mathematical manipulation for Eq. (27) yields

$$
D_{\varepsilon t}=\frac{\alpha}{\tau_{t}} \frac{\partial^{2} k_{t}}{\partial x_{j} \partial x_{j}}-\frac{2 \alpha}{\tau_{t}^{2}} \frac{\partial \tau_{t}}{\partial x_{i}} \frac{\partial k_{t}}{\partial x_{i}}-\frac{\alpha k_{t}}{\tau_{t}^{2}} \frac{\partial^{2} \tau_{t}}{\partial x_{j} \partial x_{j}}+\frac{2 \alpha k_{t}}{\tau_{t}^{3}}\left(\frac{\partial \tau_{t}}{\partial x_{i}}\right)^{2}
$$

The turbulent diffusion term of $\varepsilon_{t}, T_{\varepsilon t}$, in Eq. (16) will be modeled by the gradient transport hypothesis assuming that the turbulent transport processes parallel the molecular ones (i.e., each turbulent transport term is coupled with a molecular diffusion term of the same general form). Therefore, $T_{\varepsilon t}$ is modeled in the form

$$
T_{\varepsilon t}=-2 \frac{\alpha_{t}}{\tau_{t}^{2} \sigma_{\tau 1}} \frac{\partial k_{t}}{\partial x_{i}} \frac{\partial \tau_{t}}{\partial x_{i}}+2 \frac{\alpha_{t} k_{t}}{\tau_{t}^{3} \sigma_{\tau 2}} \frac{\partial \tau_{t}}{\partial x_{i}} \frac{\partial \tau_{t}}{\partial x_{i}}
$$

where $\sigma_{\tau 1}$ and $\sigma_{\tau 2}$ are constants. It should be mentioned here that the modeled form of the turbulent diffusion term $T_{\varepsilon t}$ in Eq. (29) is some what similar to the modeled form of the turbulent diffusion term of $\varepsilon$ used for $k-\tau$ turbulence model for velocity field $[19,20]$.

Now turning to the production and destruction terms in Eqs. (17-21). The production term $P_{\varepsilon t}^{3}$ is negligibly small in comparison with $P_{\varepsilon t}^{1}, P_{\varepsilon t}^{2}, P_{\varepsilon t}^{4}$, and $\Psi_{\varepsilon t}$ terms, therefore, it will be ignored in the proposed model. The other terms $P_{\varepsilon t}^{1}, P_{\varepsilon t}^{2}, P_{\varepsilon t}^{4}$, and $\Psi_{\varepsilon t}$ can be modeled in a way similar to the model of Nagano et al. [2] and the model of Nagano and Kim [3] as follows:

$$
\begin{aligned}
P_{\varepsilon t}^{1}+P_{\varepsilon t}^{2}+P_{\varepsilon t}^{4}-\Psi_{\varepsilon t}= & -C_{P 1} f_{P 1} \frac{\overline{u_{j} t}}{\tau_{t}} \frac{\partial \bar{T}}{\partial x_{j}}-C_{P 2} f_{P 2} \frac{k_{t}}{k \tau_{t}} \overline{u_{i} u_{j}} \frac{\partial U_{i}}{\partial x_{j}} \\
& -C_{D 1} f_{D 1} \frac{k_{t}}{\tau_{t}^{2}}-C_{D 2} f_{D 2} \frac{k_{t}}{\tau_{u} \tau_{t}}
\end{aligned}
$$

Substituting Eqs. (12), (25,26), and (28-30) into Eq. (23), without going into mathematical details here, the final modeled equation of the temperature time scale $\tau_{t}$ is given as: 


$$
\begin{aligned}
\frac{D \tau_{t}}{D t}= & \frac{\partial}{\partial x_{j}}\left[\left(\alpha+\frac{\alpha_{t}}{\sigma_{h}}\right) \frac{\partial \tau_{t}}{\partial x_{j}}\right]+\frac{2}{k_{t}}\left(\alpha+\frac{\alpha_{t}}{\sigma_{\tau 1}}\right) \frac{\partial k_{t}}{\partial x_{i}} \frac{\partial \tau_{t}}{\partial x_{i}} \\
& -\frac{2}{\tau_{t}}\left(\alpha+\frac{\alpha_{t}}{\sigma_{\tau 2}}\right) \frac{\partial \tau_{t}}{\partial x_{i}} \frac{\partial \tau_{t}}{\partial x_{i}}+\left(1-C_{P 1} f_{P 1}\right) \frac{\tau_{t} \alpha_{t}}{k_{t}}\left(\frac{\partial \bar{T}}{\partial x_{j}}\right)^{2} \\
& -C_{P 2} f_{P 2} v_{t} \frac{\tau_{t}}{k}\left(\frac{\partial U_{i}}{\partial x_{j}}\right)^{2}+C_{D 1} f_{D 1}+C_{D 2} f_{D 2} \frac{\tau_{t}}{\tau_{u}}-1
\end{aligned}
$$

In Eq. (31), $f_{P 1}, f_{P 2}, f_{D 1}$, and $f_{D 2}$ are the model functions and $C_{P 1}, C_{P 2}, C_{D 1}, C_{D 2}, \sigma_{h}, \sigma_{\tau 1}$, and $\sigma_{\tau 2}$ are the model constants. Determination of these functions and constants will be discussed later. In order to construct a rigorous two-equation heat transfer model based on the temperature variance $k_{t}$ and the temperature time scale $\tau_{t}\left(k_{t}-\tau_{t}\right.$ model $)$, it is essential to reproduce the correct behavior for near-wall features. Therefore, the wall limiting behavior of the different terms in $k_{t}$ and $\tau_{t}$ equations will be explored in the next section.

\section{NEAR-WALL LIMITING BEHAVIOR}

Viscosity and molecular conduction become dominant in the close vicinity of a stationary wall. Also, anisotropy increases due to the presence of the wall. In case of a uniform wall temperature, a fluctuating temperature near the wall may be expressed using the Taylor expansion as [3]:

$$
\begin{gathered}
t=a y+b y^{2}+c y^{3}+\ldots \\
t^{2}=a^{2} y^{2}+2 a b y^{3}+\left(2 a c+b^{2}\right) y^{4}+\ldots \\
\overline{t^{2}}=\overline{a^{2}} y^{2}+2 \overline{a b} y^{3}+\left(2 \overline{a c}+\overline{b^{2}}\right) y^{4}+\ldots
\end{gathered}
$$

where $a=a(x, z, t), b=b(x, z, t)$, and $c=c(x, z, t)$. Thus, expressions of $k_{t}$ and $\varepsilon_{t}$ in the immediate neighborhood of the wall may be given by

$$
\begin{aligned}
k_{t}=\overline{t^{2}} / 2 & =\frac{\overline{a^{2}}}{2} y^{2}+\overline{a b} y^{3}+\left(\overline{a c}+\frac{\overline{b^{2}}}{2}\right) y^{4}+\ldots \\
\varepsilon_{t} & =\alpha\left(\overline{\frac{\partial t}{\partial x_{j}} \frac{\partial t}{\partial x_{j}}}\right) \cong \alpha\left(\overline{\left(\frac{\partial t}{\partial y}\right)^{2}}\right. \\
& =\alpha \overline{a^{2}}+4 \alpha \overline{a b} y+\alpha\left(\overline{6 \overline{a c}}+4 \overline{b^{2}}\right) y^{2}+\ldots
\end{aligned}
$$

From Eq. (33) it is easily demonstrated that temperature time scale $\tau_{t}$ is given by 


$$
\tau_{t}=\frac{k_{t}}{\varepsilon_{t}}=\frac{y^{2}}{2 \alpha}-\frac{\overline{a b}}{\alpha \overline{a^{2}}} y^{3}+\ldots
$$

For velocity field, with no-slip condition at the wall, the fluctuating velocities near the wall are expanded by Taylor series around the wall as follows[19]:

$$
\begin{aligned}
& u=b_{1} y+c_{1} y^{2}+d_{1} y^{3}+\ldots \\
& v=\quad c_{2} y^{2}+d_{2} y^{3}+\ldots \\
& \mathrm{w}=b_{3} y+c_{3} y^{2}+d_{3} y^{3}+\ldots
\end{aligned}
$$

where $b_{i}=b_{i}(x, z, t), c_{i}=c_{i}(x, z, t)$, and $d_{i}=d_{i}(x, z, t)$. The application of the conitinuity equation eliminates $b_{2}$. From Eq. (35), we can easily derive the following expressions

$$
\begin{gathered}
\overline{u \mathrm{v}}=\overline{b_{1} c_{2}} y^{3}+\left(\overline{c_{1} c_{2}}+\overline{b_{1} d_{2}}\right) y^{4}+\ldots \\
k=\frac{\overline{u_{i} u_{i}}}{2}=\frac{1}{2}\left(\overline{b_{1}^{2}}+\overline{b_{3}^{2}}\right) y^{2}+\left(\overline{b_{1} c_{1}}+\overline{b_{3} c_{3}}\right) y^{3}+\ldots \\
\varepsilon=v\left(\overline{\frac{\partial u_{i}}{\partial x_{j}} \frac{\partial u_{i}}{\partial x_{j}}}\right)=v\left(\overline{b_{1}^{2}}+\overline{b_{3}^{2}}\right)+4 v\left(\overline{b_{1} c_{1}}+\overline{b_{3} c_{3}}\right) y+\ldots \\
\tau_{u}=\frac{k}{\varepsilon}=\frac{1}{2 v} y^{2}-\frac{\left(\overline{b_{1} c_{1}}+\overline{b_{3} c_{3}}\right)}{v\left(\overline{b_{1}^{2}}+\overline{b_{3}^{2}}\right)} y^{3}+\ldots
\end{gathered}
$$

The transport equation of the temperature variance $k_{t}$ used in the proposed model is rewritten as follows:

$$
\frac{D k_{t}}{D t}=D_{k t}+T_{k t}+P_{k t}-\frac{k_{t}}{\tau_{t}}
$$

From Eqs. (32-35), it is straightforward under uniform wall temperature to show that near a wall,

$$
\begin{gathered}
\overline{\mathrm{v} t}=\overline{a c_{2}} y^{3}+\left(\overline{a d_{2}}+\overline{b c_{2}}\right) y^{4}+\ldots \\
D_{k t}=\alpha \frac{\partial^{2} k_{t}}{\partial x_{j} \partial x_{j}}=\alpha \overline{a^{2}}+6 \alpha \overline{a b} y+12 \alpha\left(\overline{a c}+\frac{\overline{b^{2}}}{2}\right) y^{2}+\ldots \\
T_{k t}=\frac{\partial}{\partial x_{j}}\left(\frac{\alpha_{t}}{\sigma_{h}} \frac{\partial k_{t}}{\partial x_{j}}\right)=\left(\frac{4 \overline{a^{2}} \overline{a c_{2}}}{\sigma_{h}}\right) y^{3}+\ldots \\
P_{k t}=\alpha_{t}\left(\frac{\partial \bar{T}}{\partial x_{j}}\right)^{2}=\overline{a c_{2}} y^{3}+\left(\overline{a d_{2}}+\overline{b c_{2}}\right) y^{4}+\ldots
\end{gathered}
$$




$$
\frac{k_{t}}{\tau_{t}}=\alpha \overline{a^{2}}+4 \alpha \overline{a b} y+\alpha\left(\overline{6 \overline{a c}}+4 \overline{b^{2}}\right) y^{2}+\ldots
$$

The most essential factor that determines the character of a temperature field is the turbulent heat flux $\overline{u_{j} t}$. Accordingly, the quality of the modeling of the function $f_{\lambda}$ in equation (9), which was introduced mainly to improve the near-wall behavior of $\overline{u_{j} t}$, influences the overall quality of the proposed turbulence model. As seen from Eqs. (34), (36b), and (38a) together with Eqs. (8) and (9), the model function $f_{\lambda}$ has to satisfy $f_{\lambda} \propto y^{-1}$. This may be referred to as the condition of limiting behavior of wall turbulence. Therefore, we adopt the following function for $f_{\lambda}$ :

$$
f_{\lambda}=\left[1-\exp \left(-y^{*} / A_{\lambda}\right)\right]\left[1+B_{\lambda} / R_{h}^{0.5}\right]
$$

where $y^{*}=u_{\varepsilon} y / v$. The most important feature of the proposed $k_{t}-\tau_{t}$ heat transfer model is the introduction of the Kolmogorov velocity scale, $u_{\varepsilon}=(v \varepsilon)^{1 / 4}=\left(0.1 v k^{1.5} / L\right)^{1 / 4}$, in determining $y^{*}$ instead of using the friction velocity $u_{\tau}$, to account for the near-wall and low-Reynolds-number effects in both attached and detached flows [15]. Near the wall, we have $R_{h}=\left(k \tau_{t} / v\right)=o\left(y^{4}\right)$, so that Eq. (39) satisfies the condition of the limiting behavior of wall turbulence. Generally, the relative thickness of the conduction-dominating sublayer to that of the viscous sublayer near the wall changes with the molecular Prandtl number Pr [3]. Thus, even at the same distance from the wall $y$, the value of $f_{\lambda}$ must change according to the corresponding thickness of the conductive sublayer if the Prandtl number changes. Considering these requirements, the best values for the constants $A_{\lambda}$ and $B_{\lambda}$ are chosen as $20 / \sqrt{\operatorname{Pr}}$ and $1 / \sqrt{\operatorname{Pr}}$, respectively, for the proposed heat transfer model.

It is clearly seen from Eqs. (38b) and (38e) that the molecular diffusion and dissipation terms of $k_{t}, D_{k t}$ and $k_{t} / \tau_{t}$, respectively, balance each other at the wall only. As a corollary, to improve the near-wall region behavior, it is necessary to add a correction term $E_{k t}$ to equation (37). The term $E_{k t}$ is proposed as follows:

$$
E_{k t}=-\frac{\partial}{\partial x_{j}}\left\{f_{w k t}\left(\alpha \frac{\partial k_{t}}{\partial x_{j}}-2 \alpha f_{w k t} \frac{k_{t}}{x_{j}}\right)\right\}
$$

where $f_{w k t}$ is a damping function which is introduced so that the proposed form of $E_{k t}$ is relevant only to the wall vicinity. The damping function $f_{w k t}$ is found to be 


$$
f_{w k t}=\exp \left\{-\left(R_{h} / 5\right)^{0.5}\right\}
$$

The damping function $f_{w k t}=1.0$ at $R_{h}=0$ (at the wall) and $f_{w k t} \rightarrow 0$ away from the wall as it is obvious from Eq. (41). In the near-wall region, one can easily use Eq. (33a) to find that $E_{k t}$ in Eq. (40) as

$$
E_{k t}=-2 \alpha \overline{a b} y-6 \alpha\left(\overline{a b}+\frac{\overline{b^{2}}}{2}\right) y^{2}+\ldots
$$

Obviously, from Eqs. (38b), (38e), and (42), summation of $D_{k t}$ and $k_{t} / \tau_{t}$ exactly balances with $E_{k t}$ in the vicinity of the wall up to the first order of y. From these considerations, we finally obtain the governing equation of $k_{t}$ for the present $k_{t}-\tau_{t}$ as follows:

$$
\begin{aligned}
\frac{D k_{t}}{D t}= & \frac{\partial}{\partial x_{j}}\left\{\left(\alpha+\frac{\alpha_{t}}{\sigma_{h}}\right) \frac{\partial k_{t}}{\partial x_{j}}\right\}+\alpha\left(\frac{\partial \bar{T}}{\partial x_{j}}\right)^{2}-\frac{k_{t}}{\tau_{t}} \\
& -\frac{\partial}{\partial x_{j}}\left\{f_{w k t}\left(\alpha \frac{\partial k_{t}}{\partial x_{j}}-2 \alpha f_{w k t} \frac{k_{t}}{x_{j}}\right)\right\}
\end{aligned}
$$

The turbulent diffusion constant $\sigma_{h}$ is assigned the value of 1.0 as recommended by Nagano and Kim [3]. The eddy diffusivity for $\alpha_{t}$ is determined from Eq. (9) using the damping function $f_{\lambda}$ from Eq. (39).

We now proceed to determine the model constants and the model functions used in equation (31), $\tau_{t}$-equation. First, $C_{\lambda}$ is chosen to be 0.11 as suggested by Nagano and Kim [3], while, $C_{D 1}$ and $C_{D 2}$ are determined with reference to Abe et al.'s [15] suggestion and have the values of 2.0 and 0.9 , respectively. The constants $C_{P 1}$ and $C_{P 2}$ are to be determined with the aid of the relation for the 'constant-stress and constant-heat-flux layer' $[1-3,18]$. When the velocity field is calculated using $k-\varepsilon$ model and the temperature field is predicted using $k_{t}-\varepsilon_{t}$ model, this relation is written as:

$$
C_{D 1}-C_{P 1}=2 R\left\{C_{P 2}-C_{D 2}+\left(\kappa^{2} / \operatorname{Pr}_{t}\right) /\left(\sigma_{\phi} \sqrt{C_{\mu}}\right)\right\}
$$

Thus, substituting the standard values of $C_{\mu}=0.09$, time scale ratio $\mathrm{R}$ $\left(=\tau_{u} / \tau_{t}\right)=0.5$, turbulent Prandtl number $\operatorname{Pr}_{t}=0.9$, von Karman constant for the velocity field $\kappa=0.4$, and turbulent diffusion constant for $\varepsilon_{t}, \sigma_{\phi}=1.0$ as well as 
$C_{D 1}=2.0$ and $C_{D 2}=0.9$ as already determined into equation (44), we obtain the following relation:

$$
C_{P 2}=2.3-C_{P 1}
$$

After examining the calculated results and according to Eq. (45), the optimum values of $C_{P 1}$ and $C_{P 2}$ are 1.9 and 0.4 , respectively. It should be mentioned here that Abe et al. [15] used $C_{P 1}=1.9$ and $C_{P 2}=0.6$ in their heat transfer turbulence model. Concerning the turbulent diffusion constants $\sigma_{\tau 1}$ and $\sigma_{\tau 2}$, we set both of them to the same value of 0.6 with the aid of DNS data of Kasagi et al. [7]. By investigating equation (30), the wall limiting behavior reveals that the functions $f_{D 1}$ and $f_{D 2}$ are both proportional to $y^{2}$. These functions can be proposed in the following form:

$$
\begin{gathered}
f_{D 1}=\left\{1-\exp \left(-y^{*} / 2\right)\right\}^{2} \\
f_{D 2}=1-\exp \left[-\left(R_{h} / 5\right)^{0.5}\right\rfloor=1-f_{w k t}
\end{gathered}
$$

Also, near-wall analysis of other terms in equation (30) reveals that the generation terms can be of order $y$ and the proposed $k_{t}-\tau_{t}$ model does not suffer from any instability, though it uses the model functions of $f_{P 1}=f_{P 2}=1.0$.

In summary, our new $k_{t}-\tau_{t}$ model of turbulence is assumed to be governed by Eq. (43), $k_{t}$-equation, and Eq. (31), $\tau_{t}$-equation, along with the auxiliary relation in Eq. (9). However, in order to calculate the turbulence quantities associated with thermal field, these equations must be solved simultaneously together with the energy equation, Eq. (7). The constants and functions in the proposed two-equation heat transfer model are summarized in Table 2.

Table 2. Constants and functions in the proposed $k_{t}-\tau_{t}$ model

\begin{tabular}{|ccccc|ccccc|}
\hline $\mathrm{A}_{\lambda}$ & $\mathrm{B}_{\lambda}$ & $\mathrm{C}_{\lambda}$ & $\mathrm{C}_{\mathrm{D} 1}$ & $\mathrm{C}_{\mathrm{D} 2}$ & $\mathrm{C}_{\mathrm{P} 1}$ & $\mathrm{C}_{\mathrm{P} 2}$ & $\sigma_{h}$ & $\sigma_{\tau 1}$ & $\sigma_{\tau 2}$ \\
\hline $20 / \sqrt{\operatorname{Pr}}$ & $1 / \sqrt{\mathrm{Pr}}$ & 0.11 & 2.0 & 0.9 & 1.9 & 0.4 & 1.0 & 0.6 & 0.6 \\
\hline $\mathrm{f}_{\lambda}=\left\{1-\exp \left(-\mathrm{y}^{*} / \mathrm{A}_{\lambda}\right)\right\}\left[1+\mathrm{B}_{\lambda} / \mathrm{R}_{\mathrm{h}}{ }^{0.5}\right]$ & & \multicolumn{1}{l}{$\mathrm{f}_{\mathrm{D} 1}=\left\{1-\exp \left[-\mathrm{y}^{*} / 2\right]\right\}^{2}$} & \\
\hline $\mathrm{f}_{\mathrm{wkt}}=\exp \left\{-\left(\mathrm{R}_{\mathrm{h}} / 5\right)^{0.5}\right\}$ & & & & $\mathrm{f}_{\mathrm{D} 2}=1-\mathrm{f}_{\mathrm{wkt}}$ \\
\hline $\mathrm{f}_{\mathrm{P} 1}=1.0$ & & & $\mathrm{f}_{\mathrm{P} 2}=1.0$ \\
\hline
\end{tabular}

\section{SOLUTION PROCEDURE}

Reliable DNS databases for fully-developed two-dimensional channal flows are available at two values of $\mathrm{Re}_{\tau}$ under different wall thermal conditions, $\operatorname{Re}_{\tau}=150$ (Kasagi et al. [7]), and $\operatorname{Re}_{\tau}=180$ (Kim and Moin [6]). Therfore, in the present study, attention is focused upon these two test cases to judge the validity of the proposed two-equation heat transfer model. The governing equations are of parabolic nature and can be transformed into algebraic equivalents by using discretization 
process. A control volume finite difference procedure by Patankar [22] is used to solve these algebraic equations by using a TDMA algorithm (TriDiagonal Matrix Algorithm). The nonuniform grid technique is used in which the grid in the normal direction was given by the following formula:

$$
y_{j}=h[(j-1) /(J-1)]^{2}
$$

where $\mathrm{j}$ is the grid index, $\mathrm{h}$ is the channel half-width, and $\mathrm{J}$ is the total number of grid points in y direction. A number of 201 grid points was allocated in the computational domain to assure that the solution is independent of the grid point numbers in a normal direction.

It should be noted that for the tested channel flows, since the flow is fully developed, it is not necessary to solve the discretised equations in the stream-wise coordinate $\mathrm{x}$ and the calculations are carried out at the same location where the initial data profiles are supplied. Concerning the velocity field, the discetised equations for $\mathrm{U}, \mathrm{k}$, and $\mathrm{kL}$ are solved simultaneously in one loop in an iterative procedure. By the end of each iteration, the output results are under-relaxed to be used as initial data for the next iteration and judgement of convergence is performed. This procedure is continued until satisfying a specified convergence criterion for $\mathrm{U}$, $\mathrm{k}$, and $\mathrm{kL}$ simultaneously to get the final results for the velocity field. After that, the same procedure is applied for the discetised equations of $\bar{T}, k_{t}$, and $\tau_{t}$ to get the final results for the thermal field.

The wall boundary conditions (at $\mathrm{y}=0$ ) are: $\mathrm{U}=\mathrm{k}=\mathrm{kL}=0$ for the velocity field. For the thermal field, on the other hand, the wall thermal boundary conditions are either constant wall temperature or constant wall heat flux. The wall thermal boundary conditions are $k_{t}=\tau_{t}=0$ and the values of $\bar{T}_{w}$ or $q_{w}$ is prescribed using experimental or DNS data at the wall [16]. At the center of channel, $\partial U / \partial y=\partial k / \partial y=\partial k L / \partial y=\partial \bar{T} / \partial y=\partial k_{t} / \partial y=\partial \tau_{t} / \partial y=0$ are specified [18].

A criterion for convergence may be given by

$$
\operatorname{Max}\left|Z^{i+1} / Z^{\mathrm{i}}-1\right|<10^{-5}
$$

where $\mathrm{Z}$ stands for $\mathrm{U}, \mathrm{k}, \mathrm{kL}, \bar{T}, k_{t}$, and $\tau_{t}$. The index i denotes the number of iterations. All calculations were performed using double precision arithmetic on an IBM compatible (500 MHZ Pentium) PC computer.

\section{RESULTS AND DISCUSSION}

\subsection{Velocity Field}

For reliable evaluation of the turbulent heat transfer, it is crucial to use turbulence models which can predict both the velocity and temperature fields with high accuracy. In other words, for the accurate prediction of heat transfer in turbulent flows, we need to use turbulence models which fulfil the following requirements [15]: (1) The 
correct near-wall limiting behavior is reproduced for both the velocity and the temperature fields. (2) The temperature-field time scale is appropriately taken into account. (3) The flow fields can be simulated with sufficient accuracy.

In an earlier paper [21], the author proposed a new rigorous two-equation k-kL model of turbulence for near-wall and low-Reynolds number flows. The proposed model can reproduce correctly the near-wall limiting behavior. The proposed model has been assessed by application to fully-developed turbulent channel flows at different Reynolds numbers and flat-plate boundary layer flow. Sample of the calculated results using k-kL model is shown below in Figs. 1-6.

It is clearly seen in Figs. 1 and 2 that the overall agreement between k-kL model predictions and DNS data of Kasagi et al. [7] is excellent. The budget of kinetic energy for channel flow at $\operatorname{Re}_{\tau}=180$ is compared in Fig. 3 with DNS data of Kim et al. [23] and shows good agreement. Figure 4 shows excellent agreement between the predicted results of kinetic energy, k, using k-kL model and the DNS data of Kim [24]. The last assessment of $\mathrm{k}-\mathrm{kL}$ model mentioned here is that the case of calculation of flat-plate boundary layer flow at $\operatorname{Re}_{\theta}=1410$ as shown in Figs. 5 and 6 . The predicted results of kinetic energy and time scale of velocity field are good in accord with DNS data of Spalart [25]. In view of this, it can be concluded that the k-kL model of Youssef [21] is internally consistent and asymptotically correct as a wall is approached and, therefore, it was selected for the calculation of velocity field in the present study.

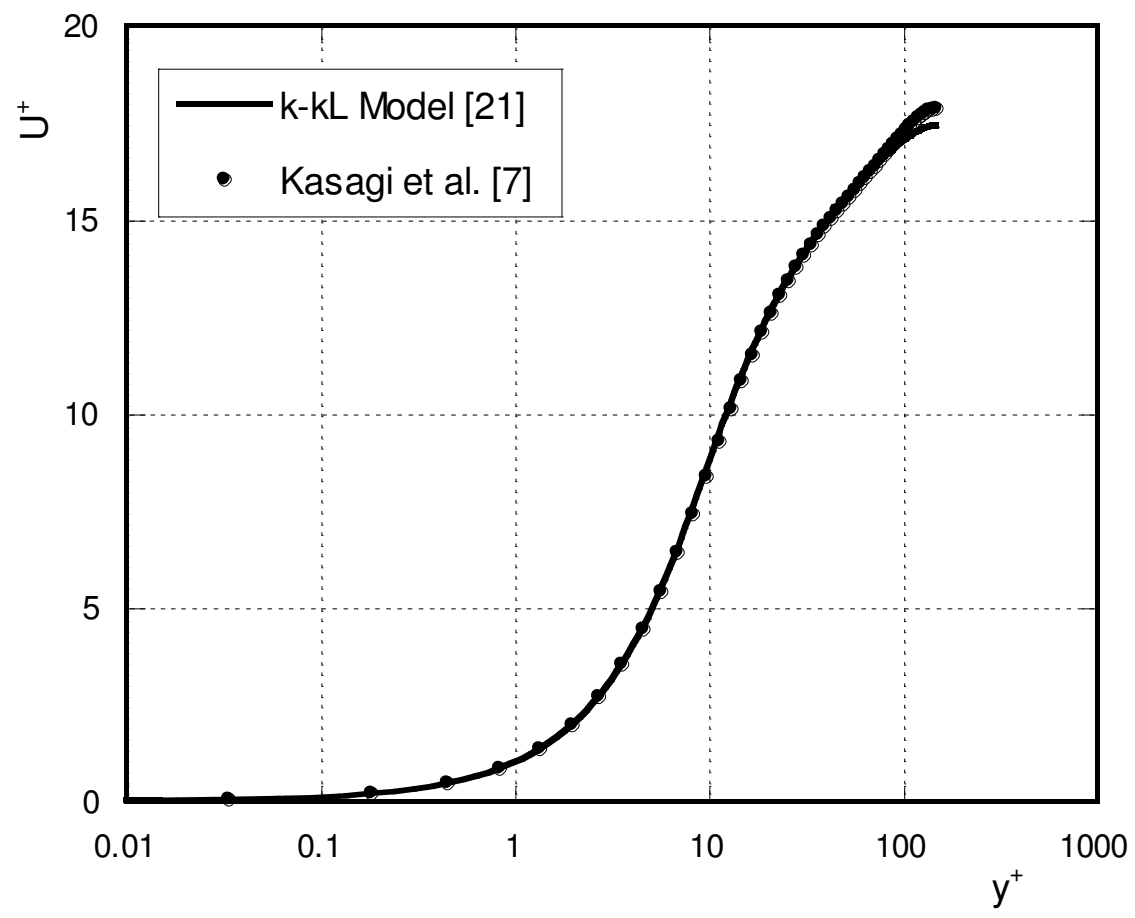

Fig. 1. Comparison of mean velocity for channel flow at $\operatorname{Re}_{\tau}=150$. 


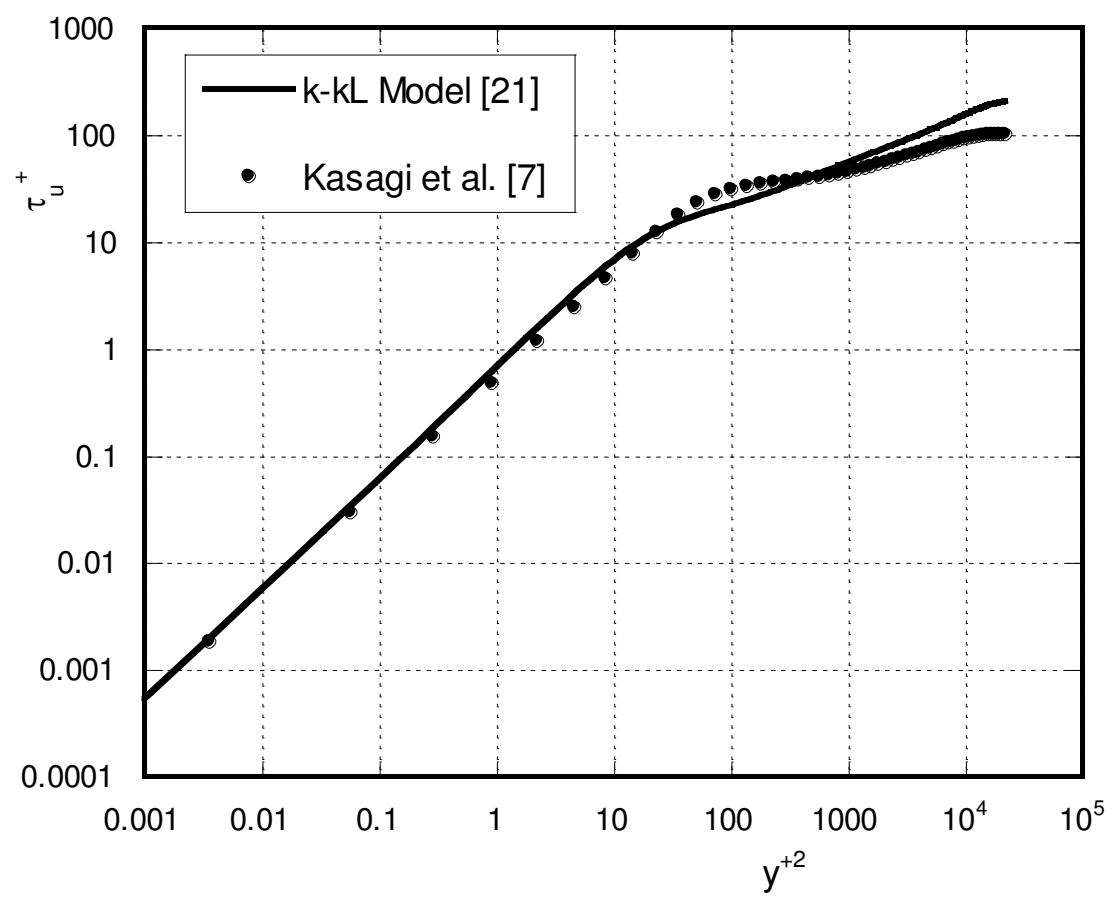

Fig. 2. Profile of time scale in channel flow at $\mathrm{Re}_{\tau}=150$.

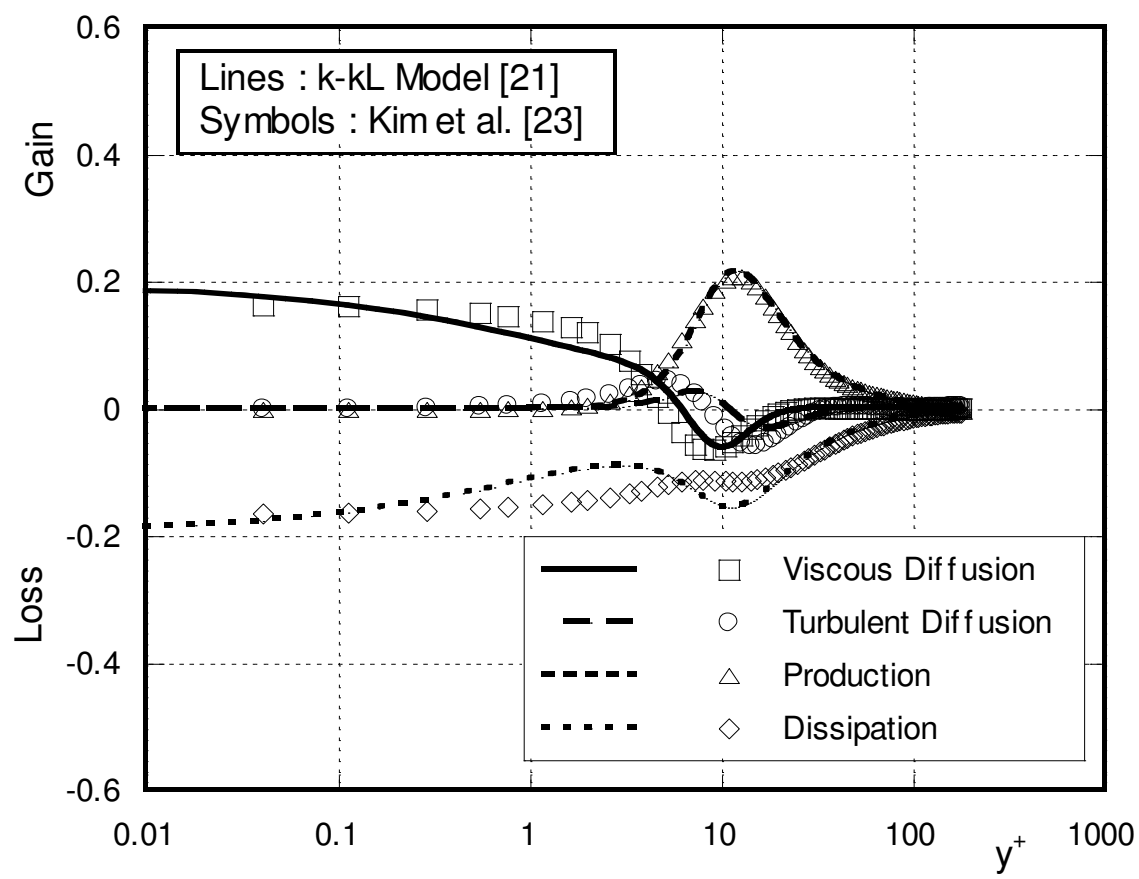

Fig. 3. Budget of kinetic energy for channel flow at $\operatorname{Re}_{\tau}=180$. 


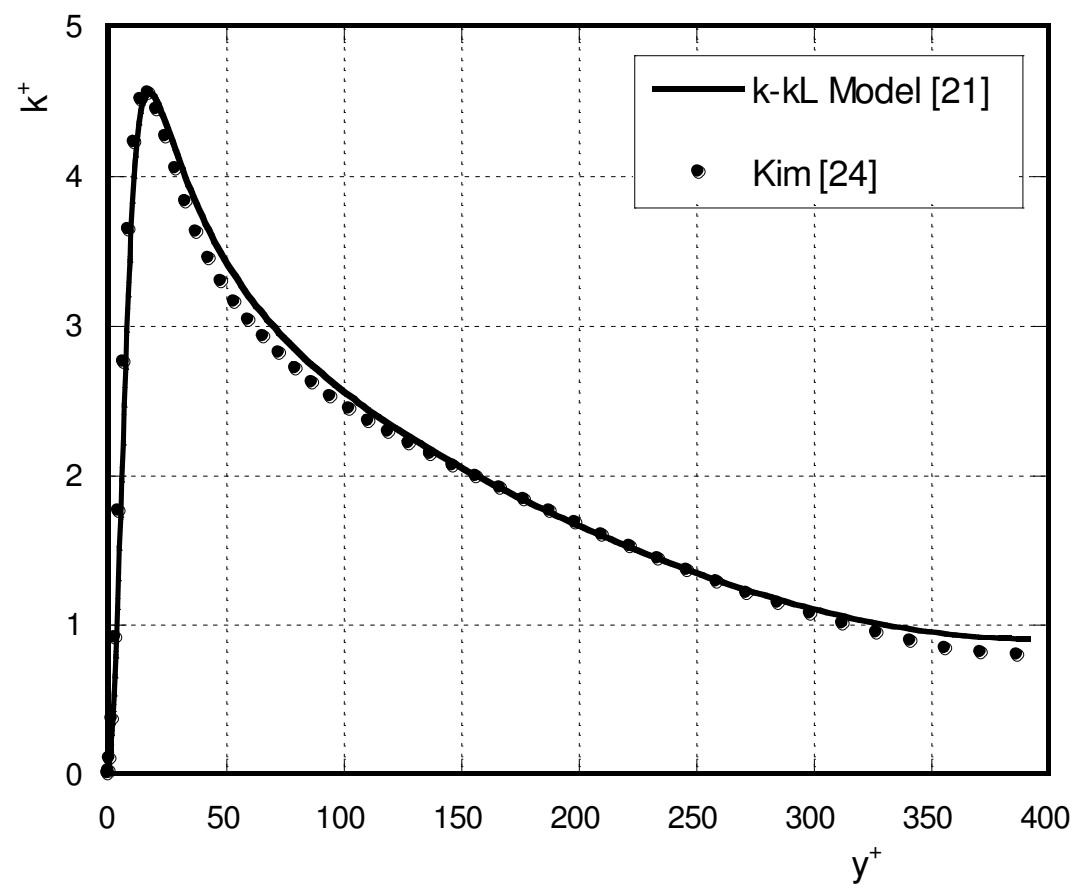

Fig. 4. Profile of kinetic energy for channel flow at $\mathrm{Re}_{\tau}=395$.

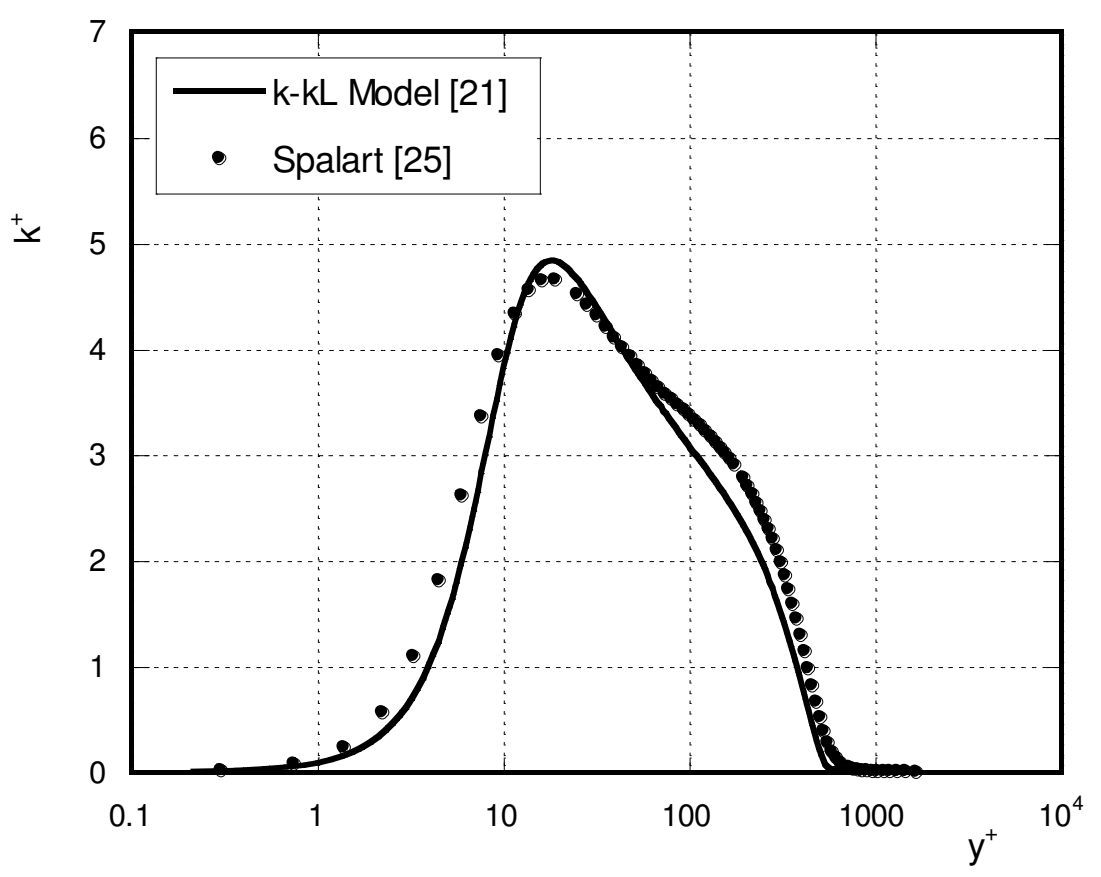

Fig. 5. Comparison of kinetic energy profile with $\mathrm{DNS}$ at $\mathrm{Re}_{\theta}=1410$. 


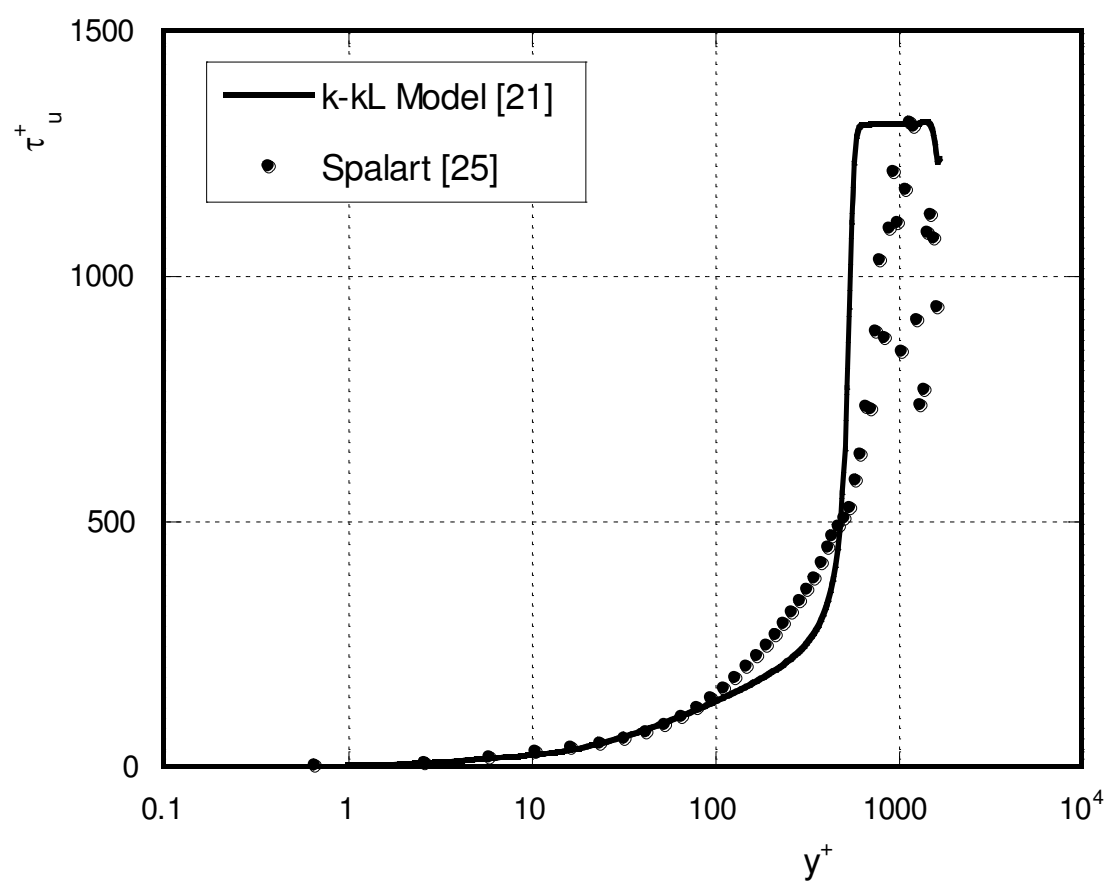

Fig. 6. Comparison of time scale profile with $\mathrm{DNS}$ at $\mathrm{Re}_{\theta}=1410$.

\subsection{Thermal Field}

Now focusing on the predicted results of the proposed $k_{t}-\tau_{t}$ model of turbulence for thermal field. Calculations of the present model are evaluated agianst direct simulation data with constant wall temperature of Kim and Moin [6] and with isoflux boundary condition of Kasagi et al. [7]. The former study is carried out at $\operatorname{Re}_{\tau}=180(\operatorname{Re}=6600)$ and a molecular Prandtl number $\operatorname{Pr}=0.71$. On the other hand, the study of Kasagi et al. [7] is carried out at $\operatorname{Re}_{\tau}=150(\operatorname{Re}=4560)$ and the same $\mathrm{Pr}$. It should be mentioned here that the DNS data of Kasagi et al. [7] have been obtained for fully-developed channel flow with isoflux wall thermal condition. However, the isoflux condition conventionally means that the time-averaged wall heatflux does not change in the streamwise direction in a two-dimensional flow. This condition is equivalent to $\partial \bar{T} / \partial x=\partial \bar{T}_{w} / \partial x=\partial \bar{T}_{m} / \partial x=$ constant. For more assessment of the present $k_{t}-\tau_{t}$ model, the present calculations of temperature field are also compared with the predictions of another two models of temperature field of type $k_{t}-\varepsilon_{t}$. These two models have been proposed by Nagano et al. [2] and Abe et al. [15]. Since the calculated velocity field properties by using k-kL model of Youssef [21] have been validated against different direct numerical simulation data, this model is used in the present study not only with the present $k_{t}-\tau_{t}$ model but also with the other two $k_{t}-\varepsilon_{t}$ models. 
The results for the isoflux turbulent channel flow case at $\operatorname{Re}_{\tau}=150(\operatorname{Re}=4560)$ and $\operatorname{Pr}=0.71$ are presented in Figs. 7-12. Mean temperature profiles are plotted in Fig. 7. In the viscous sublayer, all calculated profiles agree well with the DNS data of Kasagi et al. [7], while away from the wall, poorer performance among all model calculations is calculation with Nagano et al. [2] model. Figure 8 shows the predicted temperature variance profile compared with DNS data of Kasagi et al. [7] and with the other model calculations. It is obvious in Fig. 8 that the present prediction is in good agreement with DNS data; however, all other model calculations exhibit a discrepency with the DNS data not only in the near-wall region but also away from the wall. Examining the present model calculations, the correct near-wall limiting behavior $k_{t}^{+} \alpha y^{+2}$ is clearly emphasized as given by Eq. (33a). Figure 9 shows an acceptable agreement between the calculated results by using the present model and Nagano et al's model, while under prediction for the turbulent temperature time scale with Abe et al's model is clearly shown. The predicted results of wall-normal turbulent heat flux are compared with the DNS data in Fig. 10 and show excellent agrement except the peak with Nagano et al.'s model yields overprediction. The underprediction of temperature variance $k_{t}$ shown in Fig. 8 with Nagano et al. and Abe et al. models yiels same trend for the dissipation rate $\varepsilon_{t}$ as seen in Fig. 11. Since the budget for temperature variance is reported in ref. [7], detailed comparison is carried out for this case only and is shown in Fig. 12. What should be noticed in Fig. 12 is that the production rate balances with the dissipation rate away from the wall, while the turbulent diffusion plays an important role as the wall approached. In the viscous sublayer, the molecular diffusion and the dissipation are dominant and the general level of agreement with the DNS data of Kasagi et al. [7] is found to be excellent.

As another test case, we assess the constructed two-equation $k_{t}-\tau_{t}$ model in a turbulent channel flow with constant wall temperature case. The results provided by DNS calculation of Kim and Moin [6] at $\operatorname{Re}_{\tau}=180(\operatorname{Re}=6600)$ and a molecular Prandtl number $\operatorname{Pr}=0.71$ offers an opportunity to test the validity of the proposed model. Comparisons of the predicted mean temperature and temperature variance with DNS data of Kim and Moin [6] are shown in Figs. 13 and 14, respectively. Also, included here in these comparisons are the calculations for the $k_{t}-\varepsilon_{t}$ models of Nagano et al. [2] and Abe et al. [15]. Figure 13 shows the predicted mean temperature profile compared with the DNS data of Kim and Moin [6] and the predictions of the other $k_{t}-\varepsilon_{t}$ models. All calculated results are in perfect agreement with the DNS data in the viscous sublayer, while show underprediction in the logarithmic layer of channel. As already shown in Fig. 8, the present prediction of the temperature variance shown in Fig. 14 is in excellent agreement with DNS data; however, all other model calculations exhibit a discrepency with the DNS data not only in the near-wall region but also away from the wall. Also, what can be seen from Fig. 14 is that, the present model calculations satisfies the correct near-wall limiting behavior $k_{t}^{+} \alpha y^{+2}$ as given 
by Eq. (33a). Examining the calculated results in the present study, the proposed model is more stable than the other models since it uses the natural boundary conditions for the variables $k_{t}$ and $\tau_{t}$.

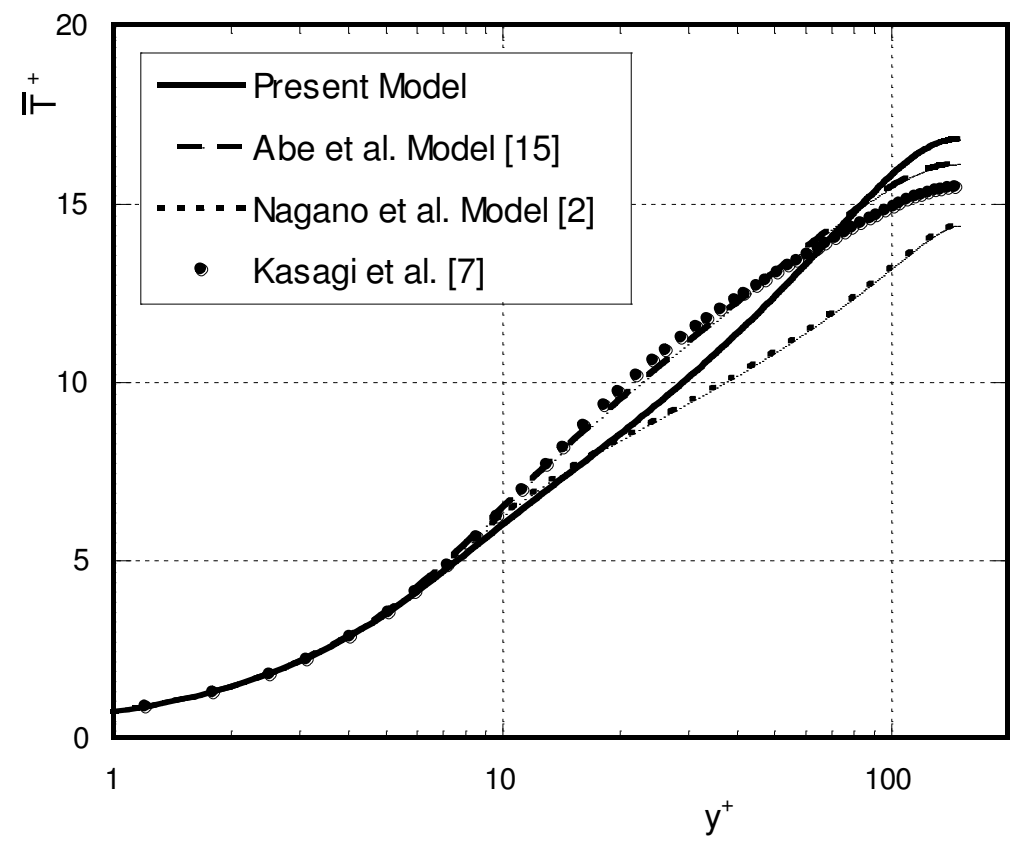

Fig. 7. Mean temperature comparison for the isoflux wall thermal case.

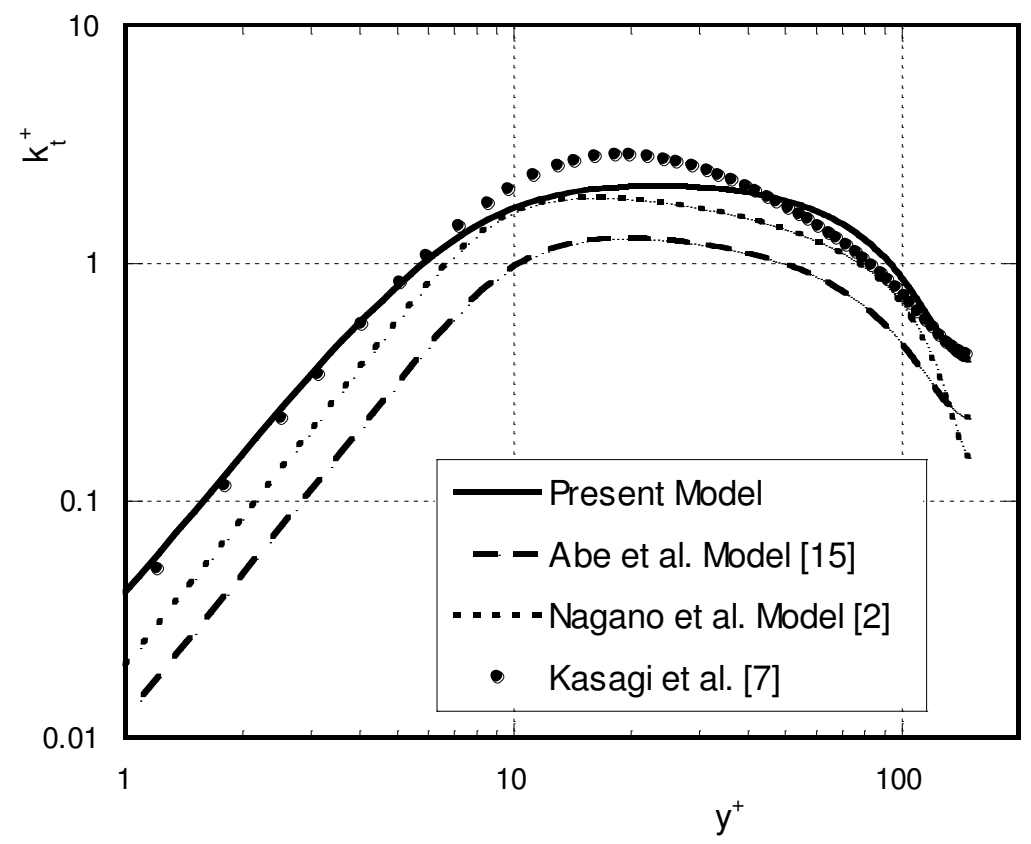

Fig. 8. Comparison of temperature variance for the isoflux wall thermal case. 


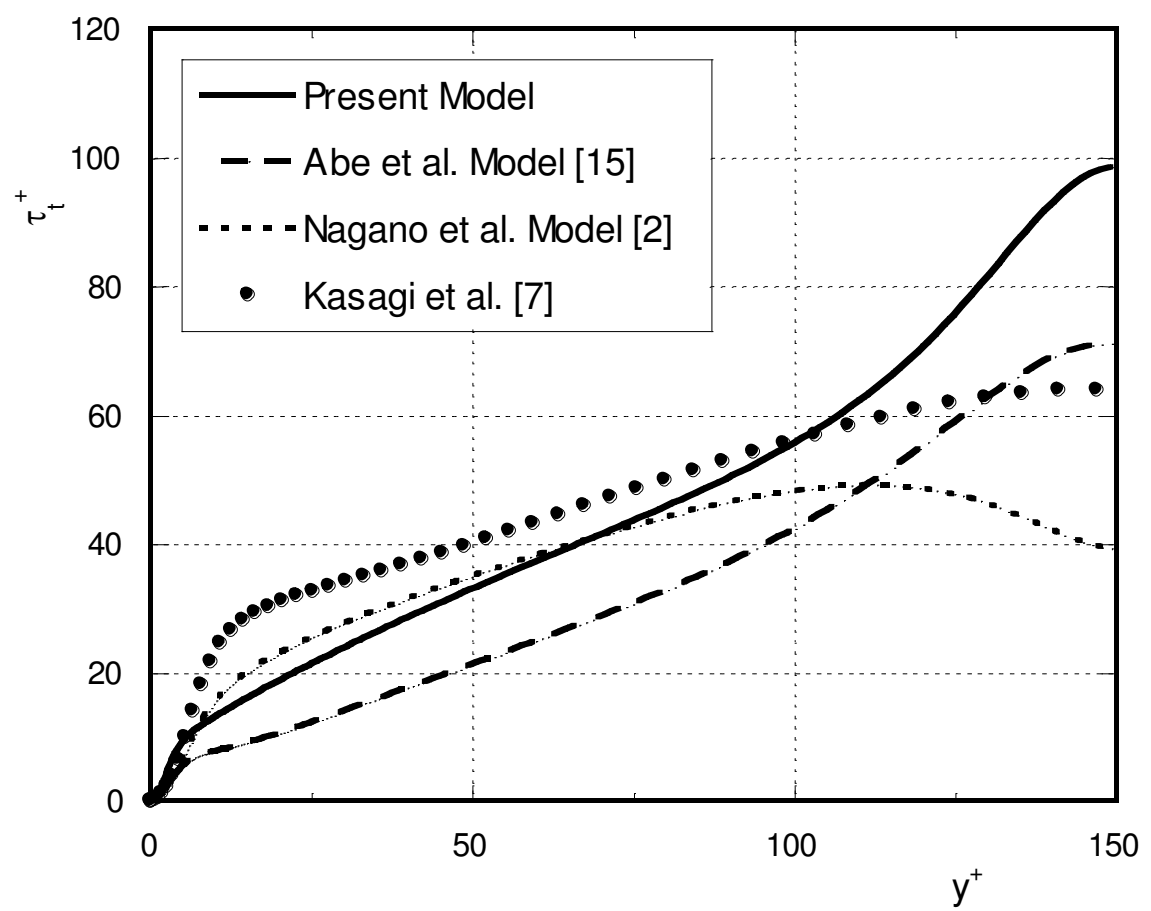

Fig. 9. Comparison of temperature time scale for the isoflux wall thermal case.

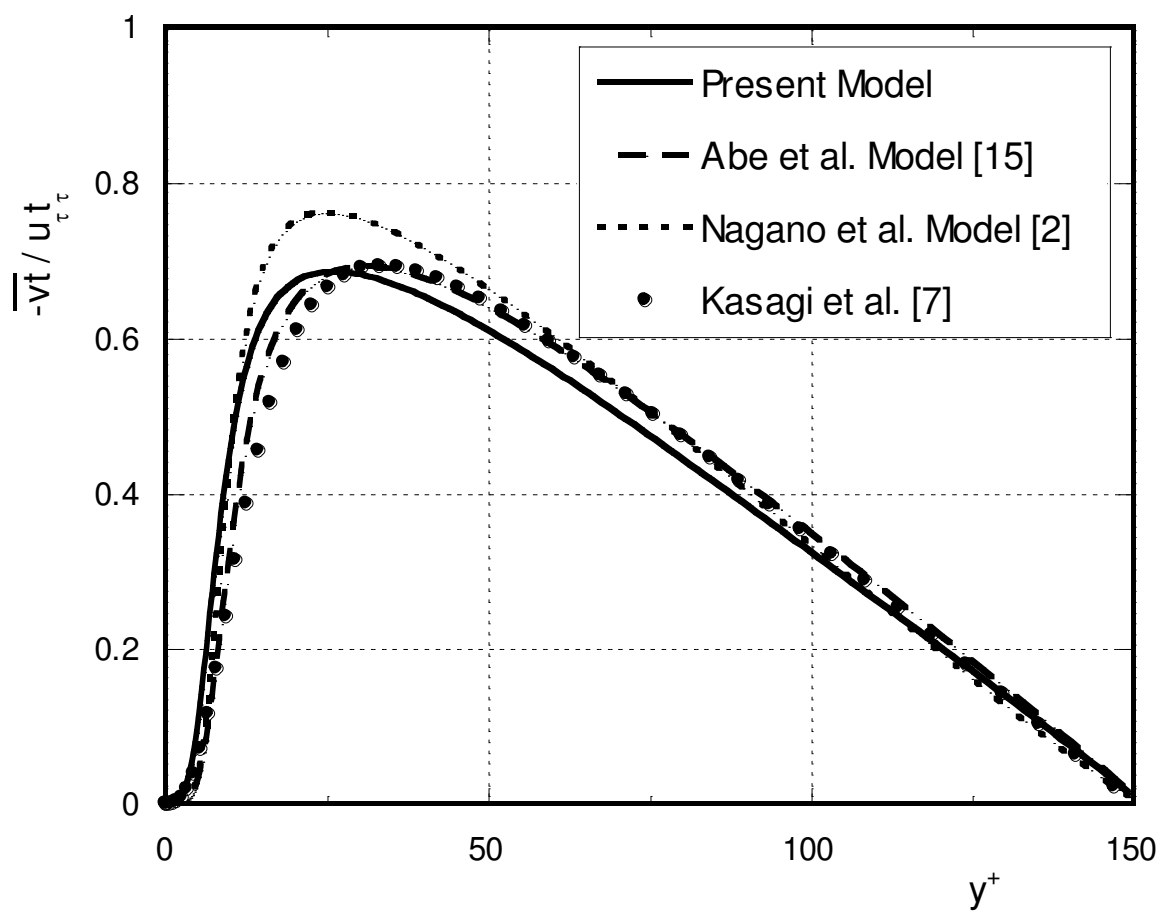

Fig. 10. Comparison of normal heat flux for the isoflux wall thermal case. 


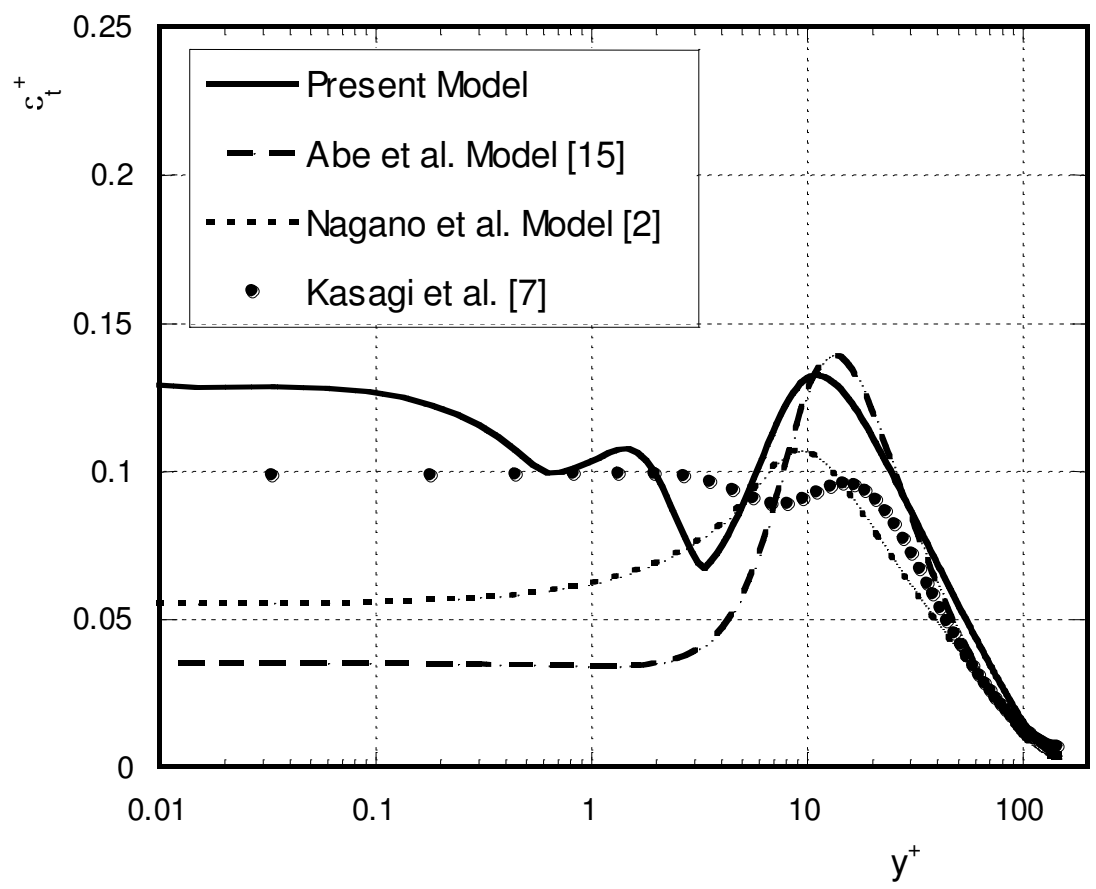

Fig. 11. Comparison of the dissipation rate of temperature variance for the isoflux wall thermal case.

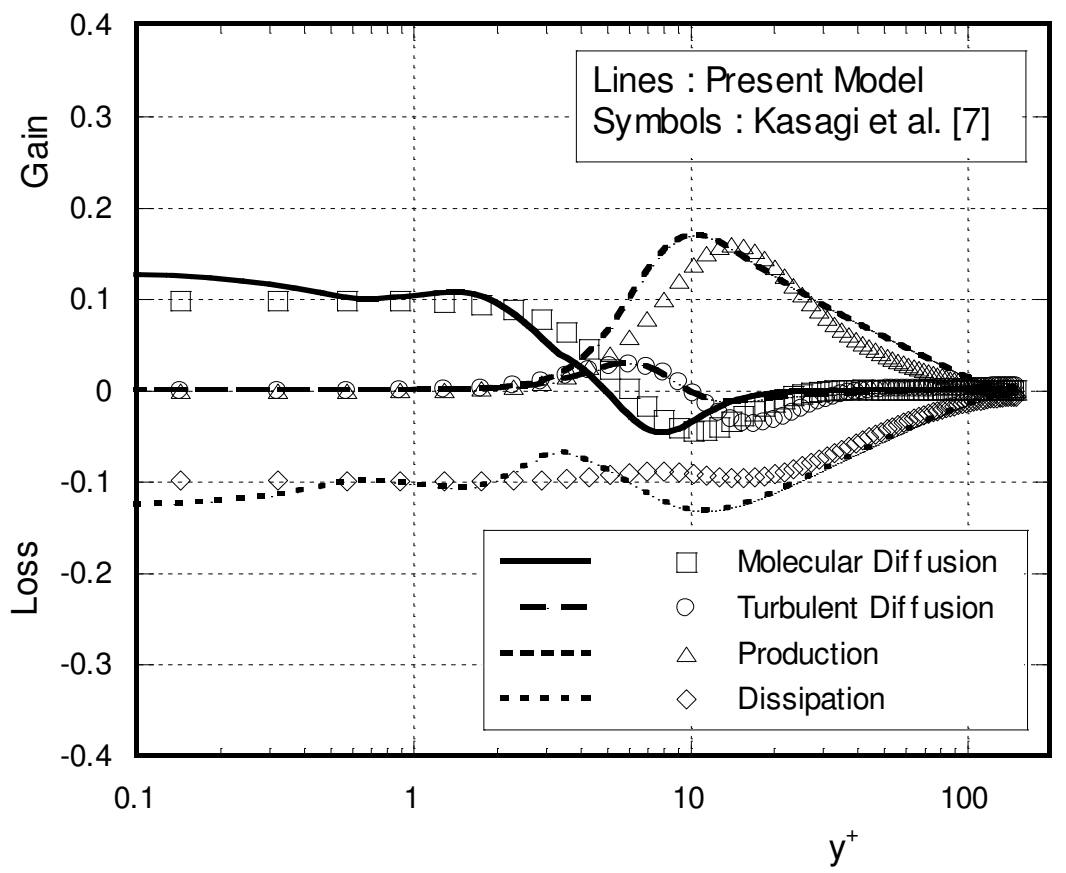

Fig. 12. Comparison of the budget of temperature variance for the isoflux wall thermal case. 


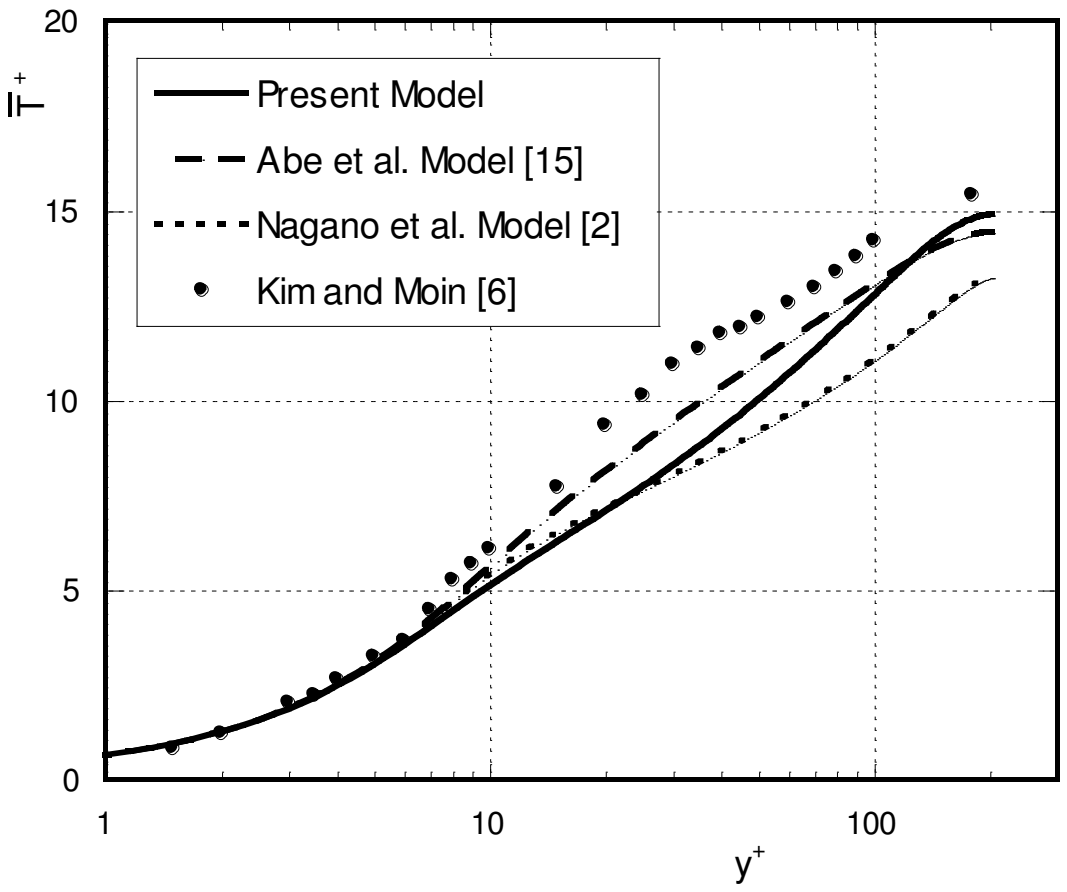

Fig. 13. Mean temperature comparison for the constant wall temperature case.

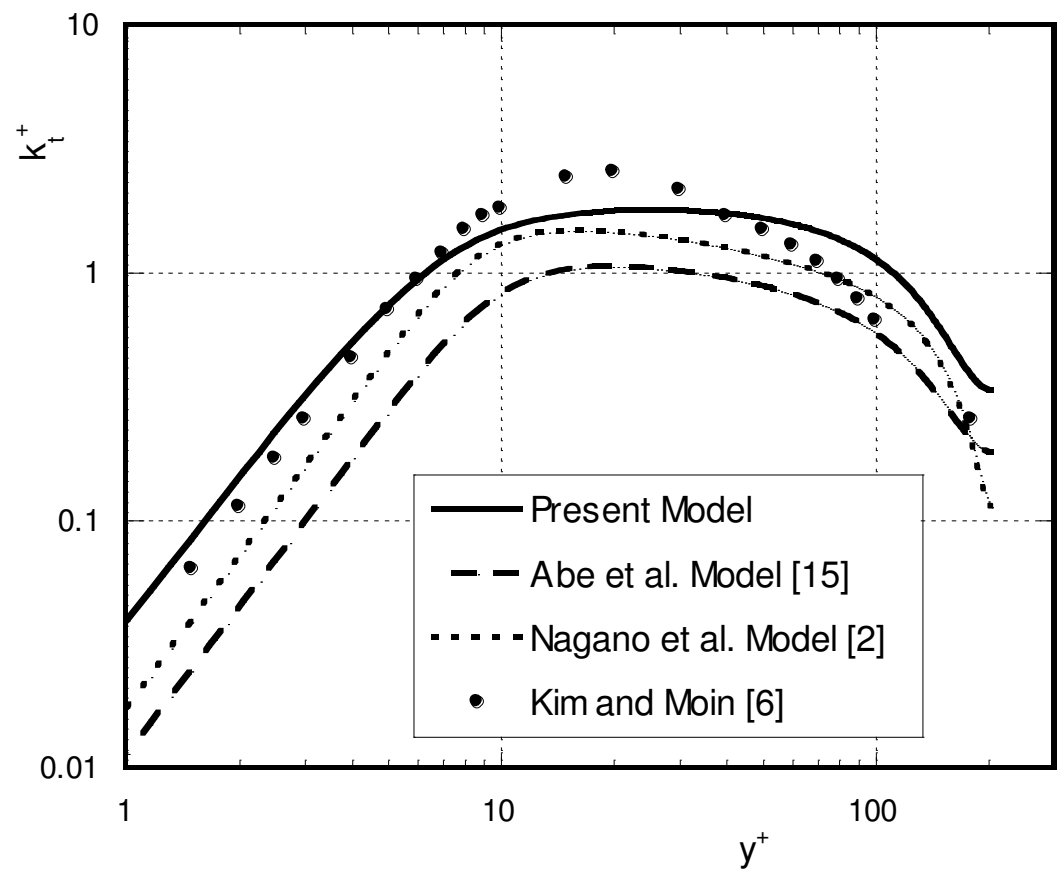

Fig. 14. Comparison of temperature variance for the constant wall temperature case. 


\section{CONCLUSIONS}

In this study, we have constructed a new rigorous two-equation heat transfer model of turbulence of type $k_{t}-\tau_{t}$. In the present model, the turbulent heat flux in the Reynolds-averaged energy equation is calculated from the eddy diffusivity for heat, $\alpha_{t}$, and the gradient time mean temperature. The eddy diffusivity for heat is proposed as function of local energy of turbulence, $\mathrm{k}$, and local temperature time scale, $\tau_{t}$, instead of using mixed time scale, $\tau_{m}$, which is composed of time scales of the velocity and temperature fields. The proposed two-equation heat transfer model solves two differential equations, one for the temperature variance, $k_{t}$, and the other for the temperature time scale, $\tau_{t}$. Since both $k_{t}$ and $\tau_{t}$ has natural boundary condition arising from the no-slip condition, the numerical stiffness problems associated with $k_{t}-\varepsilon_{t}$ models are not found. The near-wall limiting behavior of turbulent quantities associated with heat transfer has been captured with the proposed model. Therefore, an additional term is included in the temperature variance equation to enhance the prediction of near-wall behavior. Moreover, an exact and noval equation for the temperature time scale, $\tau_{t}$, is introduced. The present model is assessed by application to calculate channel flows under different wall thermal conditions with different Reynolds numbers. Comparisons between the present $k_{t}-\tau_{t}$ model, the other published $k_{t}-\varepsilon_{t}$ models, and the available DNS database were made. In all the flow cases examined, it is revealed that, strong capability of the present model in predicting accurately the DNS database, while, the other $k_{t}-\varepsilon_{t}$ models included in the comparisons failed to do so particularly in the near-wall region. Moreover, the governing equations of the present model are simple, and free of numerical stiffness. From these standpoints, the proposed model may be regarded as a two-equation heat transfer model of turbulence.

\section{REFERENCES}

[1] Youssef, M. S., Nagano, Y., and Tagawa, M., " A two-equation heat transfer model for predicting turbulent thermal fields under arbitrary wall thermal conditions", Int. J. Heat Mass Transfer 35, No. 11, pp. 3095-3104, 1992.

[2] Nagano, Y., Tagawa, M., and Tsuji, T., " An improved two-equation heat transfer model", Proc. ASME/JSME Thermal Engineering Joint Conf., 3, pp. 233-240, Reno, NV, 1991.

[3] Nagano, Y., and Kim, C., "A two-equation model for heat transport in wall turbulent shear flows", ASME J. Heat Transfer, 110, pp. 583-589, 1988.

[4] Krishnamoorthy, L. V., and Antonia, R. A., " Temperature dissipation measurements in a turbulent boundary layer", J. Fluid Mech. 176, pp. 265-281, 1987. 
[5] Krishnamoorthy, L. V., and Antonia, R. A., " Turbulent kinetic energy budget in the near-wall region", AIAA J. 26, pp. 300-302, 1987.

[6] Kim, J., and Moin, P., " Transport of passive scalars in a turbulent channel flow", In Turbulent Shear Flows 6, Springer, Berlin, pp. 85-96, 1989.

[7] Kasagi, N., Tomita, Y., and Kuroda, A., " Direct numerical simulation of passive scalar field in a turbulent channel flow", Trans ASME: J. Heat Transfer 114, pp. 598-606, 1992.

[8] Reynolds, A. J., "The prediction of turbulent Prandtl and Schmidt numbers", Int. J. Heat Mass Transfer 18, pp. 1055-1069, 1975.

[9] Nagano, Y., and Hishida, M., "Prediction and dissipation of turbulent velocity and temperature fluctuations in fully developed pipe flow", Proc. $5^{\text {th }}$ Sump. On Turbulent Shear Flows, Cornell, Ithaca, pp. 14.19-14.24, 1985.

[10] Shikazono, N., and Kasagi, N., "Second-moment closure for turbulent scalar transport at various Prandtl numbers", Int. J. Heat Mass Transfer 39, No. 14, pp. 2977-2987, 1996.

[11] Lien, F. S., and Leschziner, M. A., "Modelling 2D and 3D separation from curved surfaces with varients of second-moment closure combined with low-Re nearwall formulations", Proc. $9^{\text {th }}$ Sump. On Turbulent Shear Flows, Kyoto, pp. 13.1.1-13.1.6., 1993.

[12] Murakami, S., Mochida, A., and Ooka, R., "Numerical simulation of flow field over surface-mounted cube with various second-moment closure models", Proc. $9^{\text {th }}$ Sump. On Turbulent Shear Flows, Kyoto, pp. 13.5.1-13.5.6, 1993.

[13] Hattori, H., Nagano, Y., and Tagawa, M., " Analysis of turbulent heat transfer under various thermal conditions with two-equation models", in W. Rodi and F. Martelli (eds.), Engineering Turbulence Modelling and Eperiments 2, pp. 43-52, Elsevier, Amsterdam, 1993.

[14] Sommer, T. P., So, R. M. C., and Lai, Y. G., " A near-wall two-equation model for turbulent heat fluxes", Int. J. Heat Mass Transfer 35, pp. 3375-3387, 1992.

[15] Abe, K., Kondoh, T., and Nagano, Y., " A new turbulence model for predicting fluid flow and heat transfer in separating and reattaching flows-II. Thermal field calculations", Int. J. Heat Mass Transfer 38, pp.1467-1481, 1995.

[16] Hattori, H., and Nagano, Y., " Rigorous formulation of two-equation heat transfer model of turbulence using direct simulations", Numerical Heat Transfer 33, Part B, pp. 153-180, 1998.

[17] Sommer, T. P., So, R. M. C., and Zhang, H. S., " Supersonic flow calculations using a Reynolds-stress and a thermal eddy diffusivity turbulence model", Trans ASME: J. Fluids Engng 116, pp. 469-476,1994.

[18] Youssef, M. S., “ Near-wall modelling of turbulent flows with heat transfer", Dr. Eng. Thesis, Department of Mechanical Engineering, Nagoya Institute of Technology, Nagoya, Japan, 1994.

[19] Youssef, M. S., and Ahmed, M. A., " A new k- $\tau$ model for near-wall turbulent flows", Bulletin of the Faculty of Engineering, Assiut University 26, No. 2, July, pp. 113-124, 1998.

[20] Speziale, C. G., Abid, R., and Anderson, E. C., " Critical evaluation of two-equation models for near-wall turbulence", AIAA J. 30, No. 2, pp. 324-331, 1992. 
[21] Youssef, M. S., " A new form of k-kL turbulence model for near-wall and lowReynolds-number flows", Journal of Engineering Sciences, Assiut University, Vol. 30, No. 1, pp. 111-133, January 2002.

[22] Patankar, S. V., " Numerical Heat Transfer and Fluid Flow”, McGraw-Hill, 1980.

[23] Kim, J., Moin, P., and Moser, R., " Turbulence statistics in fully developed channel flow at low Reynolds number”, J. Fluid Mech. 177, pp. 133-166, 1987.

[24] Kim, J., "Collaborative testing of turbulence models", data disc No. 4, 1990.

[25] Spalart, P. R., " Direct simulation of a turbulent boundary layer up to $\operatorname{Re}_{\theta}=$ 1410”, J. Fluid Mech. 187, pp. 61-98, 1988.

\section{نموذج أنتقال حرارة ثنائى المعادلة لسريانات قص مضطربة ملامسة لجدار}

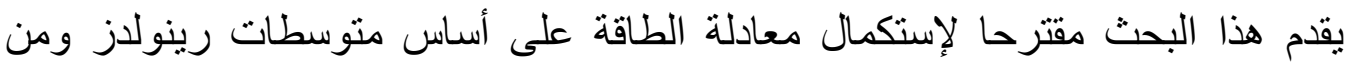

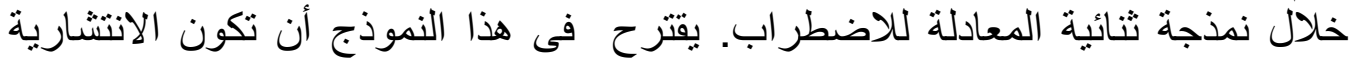

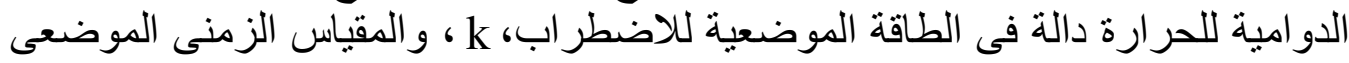

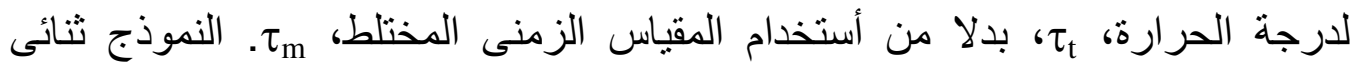

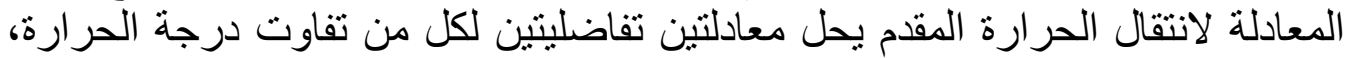

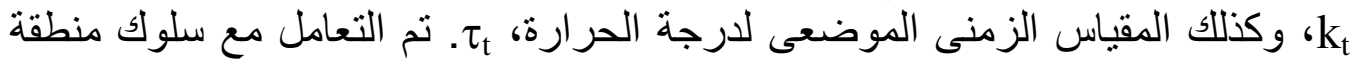

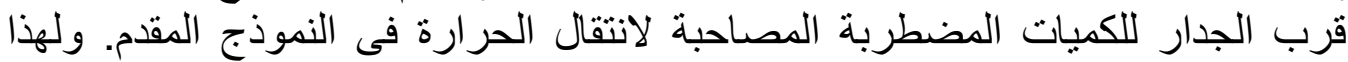

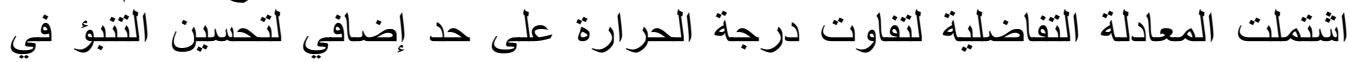
سلوك منطقة قرب الجدار. علاوة على ذللك، قدمت في الدرة اسة الفة الحالية معادلة تفاضلية

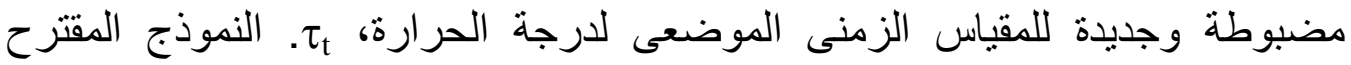

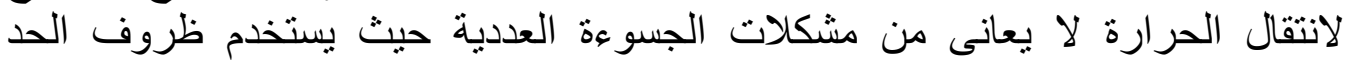

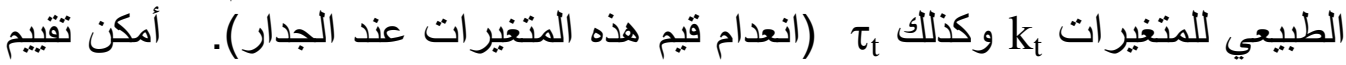

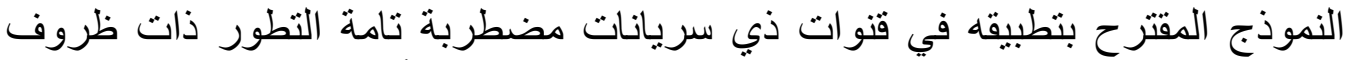

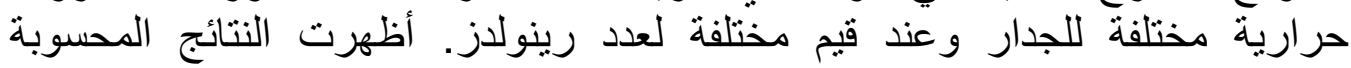
باستخدام النموذج المقدم في كل السريانات التى تم فحصها تو افقا جيدا مع نتائج المحاكاة 